\title{
JACQUET FUNCTOR AND DE CONCINI-PROCESI COMPACTIFICATION
}

\author{
NORIYUKI ABE AND YOICHI MIEDA
}

\begin{abstract}
We give a geometric realization of the Jacquet functor using a deformation of De Concini-Procesi compactification.
\end{abstract}

\section{INTRODUCTION}

The symmetric variety is used from a long time ago, when representations of a real reductive group are studied by the analytic way. On the other hand, when they are studied by the algebraic way, due to the localization theorem of BeilinsonBernstein [BB81, the flag variety is often used. However, the geometry of the symmetric space is richer than that of the flag variety. For example, the symmetric space has a boundary and one can take a "limit" to this boundary (cf. $\mathrm{KKM}^{+} 78$ ).

Fortunately, the localization theorem gives a way to realize representations as geometric objects on the symmetric variety $G / K$, where $G$ (resp. $K$ ) is the complexification of a real reductive group $G_{\mathbb{R}}$ (resp. a maximal compact subgroup $K_{\mathbb{R}}$ of $\left.G_{\mathbb{R}}\right)$. However, as far as the authors know, little is studied by such a way. In this paper, we use the symmetric variety and try to take a "limit" of such a geometric object. The limit should become the Jacquet module Cas 80 since the Jacquet module describes the asymptotic behavior of matrix coefficients HS83b. In the $p$ adic case, similar results can be found in a work of Schneider-Stuhler [SS97. They realize the Jacquet module on the boundary of the Borel-Serre compactification of the Bruhat-Tits building. The vertices of the Bruhat-Tits building for a $p$-adic semisimple group $G$ are in bijection with a union of sets of the form $G / K$, where $K$ is a maximal compact subgroup of $G$. So it can be regarded as an analogue of the symmetric variety. In this paper, we realize the Jacquet module by taking a limit on the symmetric space. Notice that, if you use the flag variety instead of the symmetric space, a realization of the Jacquet module has already been given by Emerton-Nadler-Vilonen ENV04.

We state our main results. Assume that $G_{\mathbb{R}}$ is of adjoint type. Let $G_{\mathbb{R}}=$ $K_{\mathbb{R}} A_{\mathbb{R}} N_{\mathbb{R}}$ be an Iwasawa decomposition, and $M_{\mathbb{R}}$ the centralizer of $A_{\mathbb{R}}$ in $K_{\mathbb{R}}$. Then $P_{\mathbb{R}}=M_{\mathbb{R}} A_{\mathbb{R}} N_{\mathbb{R}}$ is a Langlands decomposition of a minimal parabolic subgroup. We use lower-case fraktur letters to denote the corresponding Lie algebras and omit the subscripts " $\mathbb{R}$ " to denote complexifications. Let $X$ be the De Concini-Procesi compactification of $G / K$ DCP83. The $G$-orbit of $X$ is parameterized by a subset of $\Pi$, where $\Pi \subset \operatorname{Hom}_{\mathbb{R}}\left(\mathfrak{a}_{\mathbb{R}}, \mathbb{R}\right)$ is the set of simple restricted roots. Consider the

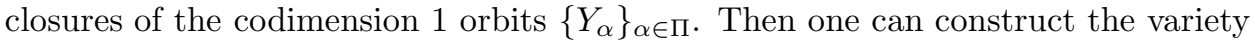
$\mathcal{X}$ over $\mathbb{A}^{\Pi}$ by iterating the deformation to the normal cone (see Section 2). The subvariety $Y_{\alpha} \subset X$ defines the subvariety $\mathcal{Y}_{\alpha} \subset \mathcal{X}$. Put $\mathcal{Z}=\mathcal{X} \backslash \bigcup_{\alpha \in \Pi} \mathcal{Y}_{\alpha}$. This

2010 Mathematics Subject Classification. 22E46, 14F05. 
is the variety which we will use. An important property of this variety is given by the following proposition. For $\Theta \subset \Pi$, let $P_{\Theta, \mathbb{R}}=M_{\Theta, \mathbb{R}} A_{\Theta, \mathbb{R}} N_{\Theta, \mathbb{R}}$ be a parabolic subgroup of $G_{\mathbb{R}}$ corresponding to $\Theta$. Put $K_{\Theta}=M_{\Theta} \cap K$. Let $f_{\mathcal{Z}}: \mathcal{Z} \rightarrow \mathbb{A}^{\Pi}$ be the canonical morphism.

Proposition 1.1 (Lemma 5.4, 5.5). For $\Theta \subset \Pi$, we have $f_{\mathcal{Z}}^{-1}\left(\left(\mathbb{G}_{m}\right)^{\Theta} \times\{0\}^{\Pi \backslash \Theta}\right) \simeq$ $G / K_{\Theta} N_{\Theta} \times\left(\mathbb{G}_{m}\right)^{\Theta}$.

Since $X$ has many orbits, it is natural to consider the "partial" Jacquet modules. Let $\mathcal{H C}_{\Theta, \rho}$ be the category of finitely generated $\left(\mathfrak{g}, K_{\Theta} N_{\Theta}\right)$-modules with the same infinitesimal characters as that of the trivial representation. For $\Theta_{2} \subset \Theta_{1} \subset \Pi$ and $V \in \mathcal{H C}_{\Theta_{1}, \rho}$, put

$$
J_{\Theta_{2}, \Theta_{1}}(V)=\left\{v \in \underset{k}{\lim _{k}} V /\left(\mathfrak{m}_{\Theta_{1}} \cap \overline{\mathfrak{n}}_{\Theta_{2}}\right)^{k} V \mid \mathfrak{n}_{\Theta_{2}}^{l} v=0 \text { for some } l\right\}
$$

where $\overline{\mathfrak{n}}_{\Theta_{2}}$ is the nilradical of the parabolic subalgebra opposite to $\mathfrak{p}_{\Theta_{2}}$. Then we can prove that $J_{\Theta_{2}, \Theta_{1}}(V) \in \mathcal{H C}_{\Theta_{2}, \rho}$ (Proposition 3.9). The usual Jacquet module is $J_{\emptyset, \Pi}$. In general, let $\operatorname{Perv}_{H}(Z)$ be the category of $H$-equivariant perverse sheaves on $Z$ for an algebraic group $H$ and a $H$-variety $Z$. Let $B$ be a Borel subgroup of $G$. The Beilinson-Bernstein correspondence and the Riemann-Hilbert correspondence give an equivalence of categories $\mathcal{H C}_{\Theta, \rho} \simeq \operatorname{Perv}_{K_{\Theta} N_{\Theta}}(G / B)$. The latter category is obviously equivalent to the category $\operatorname{Perv}_{B}\left(G / K_{\Theta} N_{\Theta}\right)$, and thus we obtain an equivalence $\mathcal{H C}_{\Theta, \rho} \simeq \operatorname{Perv}_{B}\left(G / K_{\Theta} N_{\Theta}\right)$.

Now we give our main theorem. Fix $\Theta_{2} \subset \Theta_{1} \subset \Pi$ and for each $\alpha \in \Theta_{1} \backslash \Theta_{2}$, take $n_{\alpha} \in \mathbb{Z}_{>0}$. Define $\nu: \mathbb{A}^{1} \rightarrow \mathbb{A}^{\Pi}$ by $\nu(t)=\left(0^{\Pi \backslash \Theta_{1}},\left(t^{n_{\alpha}}\right)_{\alpha \in \Theta_{1} \backslash \Theta_{2}}, 0^{\Theta_{2}}\right)$. Put $f_{\nu}: \mathcal{Z}_{\nu}=$ $\mathcal{Z} \times \times_{\mathbb{A}^{\Pi}} \mathbb{A}^{1} \rightarrow \mathbb{A}^{1}$. The by Proposition 1.1, we have $f_{\nu}^{-1}\left(\mathbb{G}_{m}\right) \simeq G / K_{\Theta_{1}} N_{\Theta_{1}} \times \mathbb{G}_{m}$ and $f_{\nu}^{-1}(0) \simeq G / K_{\Theta_{2}} N_{\Theta_{2}}$. Let $p_{\nu}: f_{\nu}^{-1}\left(\mathbb{G}_{m}\right) \simeq G / K_{\Theta_{1}} N_{\Theta_{1}} \times \mathbb{G}_{m} \rightarrow G / K_{\Theta_{1}} N_{\Theta_{1}}$ be the natural projection and $R \psi: \operatorname{Perv}\left(f_{\nu}^{-1}\left(\mathbb{G}_{m}\right)\right) \rightarrow \operatorname{Perv}\left(f_{\nu}^{-1}(0)\right)$ be the nearby cycle functor. Define the functor $\operatorname{Kat}_{\nu}: \operatorname{Perv}\left(G / K_{\Theta_{1}} N_{\Theta_{1}}\right) \rightarrow \operatorname{Perv}\left(G / K_{\Theta_{2}} N_{\Theta_{2}}\right)$ by $\mathrm{Kat}_{\nu}=R \psi \circ p_{\nu}^{*}$.

Theorem 1.2. We have the following commutative diagram:

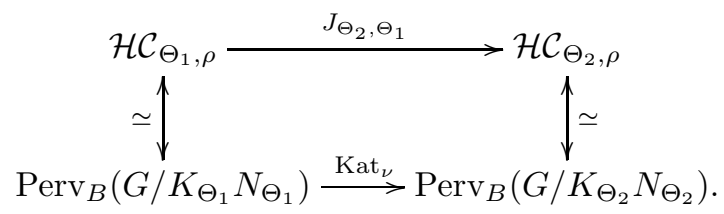

We summarize the contents of this paper. In Section 2, we give preliminaries on the deformation to the normal cone. The definition and the properties of the partial Jacquet functor $J_{\Theta_{2}, \Theta_{1}}$ are given in Section 3 . We review the theorem of Emerton-Nadler-Vilonen in Section 4 . We will use their result to prove our theorem. We finish a proof of the main theorem in Section 5 .

\section{ACKNOWLEDGMENT}

We thank Syu Kato for giving us the construction when $G_{\mathbb{R}}=\mathrm{PGL}_{2}(\mathbb{R})$.

\section{Deformation to normal CONE}

Let $X$ be a scheme of finite type over $\mathbb{C}$ and $Y$ its closed subscheme. Then we can construct a family $f: \mathcal{X} \rightarrow \mathbb{A}^{1}$, called the deformation to the normal cone, that satisfies the following: 
- over $\mathbb{G}_{m}$, it is a constant family $X \times \mathbb{G}_{m} \rightarrow \mathbb{G}_{m}$, and

- the fiber $f^{-1}(0)$ at 0 is isomorphic to the normal cone $C_{Y}(X)$.

Recall that, if we write $\mathcal{I}$ for the defining ideal of $Y \subset X$, the normal cone $C_{Y}(X)$ is the scheme $\operatorname{Spec} \bigoplus_{k=0}^{\infty} \mathcal{I}^{k} / \mathcal{I}^{k+1}$ over $Y$. If $X$ and $Y$ are smooth over $\mathbb{C}, C_{Y}(X)$ is isomorphic to the normal bundle $T_{Y}(X)$.

Let us recall briefly its construction. For more detail, see [Ful98, Chapter 5]. Let $\widetilde{\mathcal{X}}$ be the blow-up of $X \times \mathbb{A}^{1}$ along $Y \times\{0\}$. Then, by the universal property of the blow-up, we have a natural morphism $X \times\{0\} \rightarrow \widetilde{\mathcal{X}}$, which is a closed immersion. We define $\mathcal{X}$ as the complement of its image in $\tilde{\mathcal{X}}$, and $f: \mathcal{X} \rightarrow \mathbb{A}^{1}$ as the composite of $\mathcal{X} \hookrightarrow \widetilde{\mathcal{X}} \rightarrow X \times \mathbb{A}^{1} \rightarrow \mathbb{A}^{1}$. If $X$ is an affine scheme Spec $A$, we may describe $\mathcal{X}$ more explicitly as follows. Let $I$ be the defining ideal of $Y \subset X$. Then $\mathcal{X}=\operatorname{Spec} \bigoplus_{n \in \mathbb{Z}} I^{-n} T^{n}$, where $T$ is an indeterminate such that $\mathbb{A}^{1}=\operatorname{Spec} \mathbb{C}[T]$. Note that we set $I^{n}=A$ for a negative integer $n$, and regard $\bigoplus_{n \in \mathbb{Z}} I^{-n} T^{n}$ as a subring of the Laurent polynomial ring $A\left[T^{ \pm 1}\right]$.

To any subscheme $Z$ of $X$, we can attach a subscheme $\mathcal{Z}$ of $\mathcal{X}$ such that $\mathcal{Z} \rightarrow \mathbb{A}^{1}$ is the deformation to the normal cone with respect to $Y \cap Z \subset Z$. If $Z$ is open in $X$, then $\mathcal{Z}$ is simply the inverse image of $Z \times \mathbb{A}^{1}$ under $\mathcal{X} \rightarrow X \times \mathbb{A}^{1}$. On the other hand, if $Z$ is closed in $X$, then $\mathcal{Z}$ is the strict transform of $Z \times \mathbb{A}^{1} \subset X \times \mathbb{A}^{1}$ in $\mathcal{X}$; namely, $\mathcal{Z}$ is the closure of $f^{-1}\left(Z \times \mathbb{G}_{m}\right)$ in $\mathcal{X}$.

For our purpose, iteration of this construction is important. Now let $X$ be a scheme which is smooth of finite type over $\mathbb{C}, Y$ its effective divisor, and $\bigcup_{i=1}^{l} Y_{i}$ the irreducible decomposition of $Y$. Assume that $Y$ is a strict normal crossing divisor. Namely, for each subset $\Theta \subset\{1, \ldots, l\}$, we assume that $Y_{\Theta}=\bigcap_{i \in \Theta} Y_{i}$ is smooth over $\mathbb{C}$. It is equivalent to saying that $Y \subset X$ is étale locally isomorphic to $\left(T_{1} \cdots T_{l}=0\right) \subset \mathbb{A}^{n}=\operatorname{Spec} \mathbb{C}\left[T_{1}, \ldots, T_{n}\right]$ and every irreducible component of $Y$ is smooth over $\mathbb{C}$. Under this setting, let $\mathcal{X}^{(1)} \rightarrow \mathbb{A}^{1}$ be the deformation to the normal cone with respect to $Y_{1} \subset X$. For each $i$, the closed subscheme $Y_{i}$ of $X$ induces a closed subscheme $\mathcal{Y}_{i}^{(1)}$ of $\mathcal{X}^{(1)}$. Next, consider the deformation to the normal cone $\mathcal{X}^{(2)} \rightarrow \mathbb{A}^{1}$ with respect to $\mathcal{Y}_{2}^{(1)} \subset \mathcal{X}^{(1)}$ and closed subschemes $\mathcal{Y}_{i}^{(2)}$. Inductively, we can define a family $\mathcal{X}^{(k)} \rightarrow \mathbb{A}^{1}$ and closed subschemes $\mathcal{Y}_{i}^{(k)}$ of $\mathcal{X}^{(k)}$. Recall that, by construction, $\mathcal{X}^{(k)}$ is equipped with a natural structure morphism $\mathcal{X}^{(k)} \rightarrow \mathcal{X}^{(k-1)} \times \mathbb{A}^{1}$, where $\mathcal{X}^{(k)} \rightarrow \mathbb{A}^{1}$ is the composite of it with the second projection (here we put $\mathcal{X}^{(0)}=X$ ). Therefore, we get a natural morphism $\pi_{k}: \mathcal{X}^{(k)} \rightarrow X \times \mathbb{A}^{k}$ and $f_{k}=\mathrm{pr}_{2} \circ \pi: \mathcal{X}^{(k)} \rightarrow \mathbb{A}^{k}$. If $k=l$, we simply write $\mathcal{X}, \mathcal{Y}_{i}$, $\pi, f$ for $\mathcal{X}^{(l)}, \mathcal{Y}_{i}^{(l)}, \pi_{l}, f_{l}$, respectively.

First let us consider étale locally. Assume that $X=\mathbb{A}^{n}=\operatorname{Spec} \mathbb{C}\left[S_{1}, \ldots, S_{n}\right]$ and $Y_{i}$ is given by the equation $S_{i}=0$ for each $i$. Then we have

$$
\begin{aligned}
\mathcal{X}^{(1)} & =\operatorname{Spec} \mathbb{C}\left[\frac{S_{1}}{T_{1}}, S_{2}, \ldots, S_{n}, T_{1}\right], \quad \mathcal{Y}_{i}^{(1)}: \frac{S_{i}}{T_{i}}=0(i=1), S_{i}=0(i \geq 2), \\
\mathcal{X}^{(2)} & =\operatorname{Spec} \mathbb{C}\left[\frac{S_{1}}{T_{1}}, \frac{S_{2}}{T_{2}}, S_{3}, \ldots, S_{n}, T_{1}, T_{2}\right], \quad \mathcal{Y}_{i}^{(2)}: \frac{S_{i}}{T_{i}}=0(i \leq 2), S_{i}=0(i \geq 3), \\
& \vdots \\
\mathcal{X}^{(l)} & =\operatorname{Spec} \mathbb{C}\left[\frac{S_{1}}{T_{1}}, \ldots, \frac{S_{l}}{T_{l}}, S_{l+1}, \ldots, S_{n}, T_{1}, \ldots, T_{l}\right], \quad \mathcal{Y}_{i}^{(l)}: \frac{S_{i}}{T_{i}}=0 .
\end{aligned}
$$

This computation can be generalized to the case where $X$ is affine: 
Lemma 2.1. Assume that $X$ is an affine scheme $\operatorname{Spec} A$. Let $I_{i}$ be the defining ideal of $Y_{i}$. Then, we have

$$
\mathcal{X}=\operatorname{Spec} \bigoplus_{\underline{n} \in \mathbb{Z}^{l}} I^{-\underline{n}} T^{\underline{n}},
$$

where $I^{-\underline{n}}=I_{1}^{-n_{1}} \cdots I_{l}^{-n_{l}}$ and $T^{\underline{n}}=T_{1}^{n_{1}} \cdots T_{l}^{n_{l}}$ for $\underline{n}=\left(n_{1}, \ldots, n_{l}\right) \in \mathbb{Z}^{l}$.

Proof. As explained above, we have $\mathcal{X}^{(1)}=\operatorname{Spec} B^{(1)}$ for $B^{(1)}=\bigoplus_{n_{1} \in \mathbb{Z}} I_{1}^{-n_{1}} T_{1}^{n_{1}}$. The local calculation tells us that the defining ideal of $\mathcal{Y}_{2}^{(1)}$ is $I_{2} B^{(1)}$. Thus we have $\mathcal{X}^{(2)}=\operatorname{Spec} B^{(2)}$ for $B^{(2)}=\bigoplus_{n_{2} \in \mathbb{Z}} I_{2}^{-n_{2}} B^{(1)} T_{2}^{n_{2}}=\bigoplus_{\left(n_{1}, n_{2}\right) \in \mathbb{Z}^{2}} I_{1}^{-n_{1}} I_{2}^{-n_{2}} T_{1}^{n_{1}} T_{2}^{n_{2}}$. We can proceed similarly to obtain the desired formula.

Remark 2.2. By the lemma above, we know that $\mathcal{X}$ is independent of the labeling of $Y_{1}, \ldots, Y_{l}$.

For each subset $\Theta$ of $\{1, \ldots, l\}$, set $\mathcal{X}_{\Theta}=f^{-1}\left(\left(\mathbb{G}_{m}\right)^{\Theta} \times\{0\}^{\Theta^{c}}\right)$, where $\Theta^{c}=$ $\{1, \ldots, l\} \backslash \Theta$. Recall that we put $Y_{\Theta}=\bigcap_{i \in \Theta} Y_{i}$.

Lemma 2.3. We have $\mathcal{X}_{\Theta} \simeq C_{Y_{\Theta c}}(X) \times\left(\mathbb{G}_{m}\right)^{\Theta}$.

Proof. We may assume that $X$ is an affine scheme $\operatorname{Spec} A$. Let $I_{i}$ be the defining ideal of $Y_{i}$ and put $B=\bigoplus_{\underline{n} \in \mathbb{Z}^{l}} I^{-\underline{n}} T^{\underline{n}}$. By Lemma 2.1, we have

$$
\mathcal{X}_{\Theta}=\operatorname{Spec} B\left[T_{i}^{-1} \mid i \in \Theta\right] /\left(T_{i} \mid i \in \Theta^{c}\right),
$$

where $\left(T_{i} \mid i \in \Theta^{c}\right)$ is the ideal generated by $T_{i}$ for $i \in \Theta^{c}$. Note that $B\left[T_{i}^{-1} \mid i \in\right.$ $\Theta]$ is isomorphic to $\left(\bigoplus_{\underline{n} \in \mathbb{Z}^{\Theta^{c}}} I^{-\underline{n}} T^{\underline{n}}\right) \otimes_{\mathbb{C}} \mathbb{C}\left[T_{i}^{ \pm 1} \mid i \in \Theta\right]$. Therefore, by replacing $\left\{Y_{1}, \ldots, Y_{l}\right\}$ with $\left\{Y_{i} \mid i \in \Theta^{c}\right\}$, we may assume that $\Theta=\emptyset$.

Put $J=I_{1}+\cdots+I_{l}$. By the definition, we have

$$
\mathcal{X}_{\emptyset}=\operatorname{Spec} B /\left(T_{1}, \ldots, T_{l}\right)=\operatorname{Spec} \bigoplus_{\underline{n} \in\left(\mathbb{Z}_{\geq 0}\right)^{l}}\left(I^{\underline{n}} / J I^{\underline{n}}\right) T^{-\underline{n}}=\operatorname{Spec} \bigoplus_{\underline{n} \in\left(\mathbb{Z}_{\geq 0}\right)^{l}} I^{\underline{n}} / J I^{\underline{n}} .
$$

On the other hand, we have $C_{Y_{\{1, \ldots, l\}}}(X)=\operatorname{Spec} \bigoplus_{k=0}^{\infty} J^{k} / J^{k+1}$. Therefore we have a natural morphism $C_{Y_{\{1, \ldots, l\}}}(X) \rightarrow \mathcal{X}_{\emptyset}$ by sending $I^{\underline{n}} / J I^{\underline{n}}$ to $J^{k} / J^{k+1}$ where $k=n_{1}+\cdots+n_{l}$. By étale local calculation, it is easily seen that this morphism is an isomorphism.

Lemma 2.4. Assume that $X=X^{\prime} \times \mathbb{A}^{l}$ and $Y_{i}=\left\{\left(x,\left(c_{j}\right)\right) \in X \mid c_{i}=0\right\}$.

(1) We have an isomorphism $\mathcal{X} \simeq X^{\prime} \times \mathbb{A}^{l} \times \mathbb{A}^{l}$ under which $\pi: \mathcal{X} \rightarrow X \times \mathbb{A}^{l}=$ $X^{\prime} \times \mathbb{A}^{l} \times \mathbb{A}^{l}$ is given by $\left(x,\left(d_{i}\right),\left(t_{i}\right)\right) \mapsto\left(x,\left(d_{i} t_{i}\right),\left(t_{i}\right)\right)$.

(2) The projection $\mathcal{X}_{\Theta} \simeq C_{Y_{\Theta c}}(X) \times\left(\mathbb{G}_{m}\right)^{\Theta} \rightarrow C_{Y_{\Theta c}}(X) \rightarrow Y_{\Theta^{c}}$ is given by $\left(x,\left(d_{i}\right),\left(t_{i}\right)\right) \mapsto\left(x,\left(d_{i} t_{i}\right)\right)$.

(3) Let $G$ be an algebraic group over $\mathbb{C}$. Assume that we are given an action of $G$ on $X^{\prime}$ and characters $\chi_{i}: G \rightarrow \mathbb{G}_{m}$. These induce an action of $G$ on $X$ by $\left(x,\left(c_{i}\right)\right) \rightarrow\left(g x,\left(\chi_{i}(g) c_{i}\right)\right)$ which preserves $Y_{i}$. Then, the induced action on $\mathcal{X} \simeq X^{\prime} \times \mathbb{A}^{l} \times \mathbb{A}^{l}$ is given by $\left(x,\left(d_{i}\right),\left(t_{i}\right)\right) \mapsto\left(g x,\left(\chi_{i}(g) d_{i}\right),\left(t_{i}\right)\right)$.

Proof. (1) Since our construction clearly commutes with a smooth base change, we may assume that $X^{\prime}=\operatorname{Spec} \mathbb{C}$. Then the local calculation above gives us the desired isomorphism (we have only to take $n=l$ ).

(2) In the same way as in (1), we may assume that $X^{\prime}=\operatorname{Spec} \mathbb{C}$ and use the local calculation. 
(3) Since $\pi: \mathcal{X} \rightarrow X \times \mathbb{A}^{l}$ is $G$-equivariant, for $\left(x,\left(d_{i}\right),\left(t_{i}\right)\right) \in \mathcal{X}$, we have $\pi\left(g\left(x,\left(d_{i}\right),\left(t_{i}\right)\right)\right)=g\left(x,\left(d_{i} t_{i}\right),\left(t_{i}\right)\right)=\left(g x,\left(\chi_{i}(g) d_{i} t_{i}\right),\left(t_{i}\right)\right)=\pi\left(g x,\left(\chi_{i}(g) d_{i}\right),\left(t_{i}\right)\right)$. On the other hand, $\pi$ is an isomorphism over the open subset $X \times\left(\mathbb{G}_{m}\right)^{l} \subset X \times \mathbb{A}^{l}$. Therefore, on the dense open subset $\pi^{-1}\left(X \times\left(\mathbb{G}_{m}\right)^{l}\right)$ of $\mathcal{X}$, the action of $G$ is given by $\left(x,\left(d_{i}\right),\left(t_{i}\right)\right) \mapsto\left(g x,\left(\chi_{i}(g) d_{i}\right),\left(t_{i}\right)\right)$. Hence it is given by the same formula over the whole $\mathcal{X}$.

\section{The JaCquet FunCtors}

In this section, we recall some preliminaries on the Jacquet modules, which are well-known. (For example, some of them are proved in [HS83b, Wal88.) However, we give proofs for the sake of completeness.

Let $G_{\mathbb{R}}$ be a connected reductive linear algebraic group over $\mathbb{R}, G_{\mathbb{R}}=K_{\mathbb{R}} A_{\mathbb{R}} N_{\mathbb{R}}$ an Iwasawa decomposition, and $M_{\mathbb{R}}$ the centralizer of $A_{\mathbb{R}}$ in $K_{\mathbb{R}}$. Then $P_{\mathbb{R}}=$ $M_{\mathbb{R}} A_{\mathbb{R}} N_{\mathbb{R}}$ is a Langlands decomposition of a minimal parabolic subgroup. We use lower-case fraktur letters to denote the corresponding Lie algebras and omit the subscripts " $\mathbb{R}$ " to denote complexifications. Fix a Cartan involution $\theta$ such that $K=\{g \in G \mid \theta(g)=g\}$. Let $\Sigma$ be the restricted root system for $(\mathfrak{g}, \mathfrak{a})$ and $\Sigma^{+}$the positive system corresponding to $\mathfrak{n}$. Then $\Sigma^{+}$determines the set of simple roots $\Pi \subset \Sigma^{+}$. As usual, the universal enveloping algebra of $\mathfrak{g}$ is denoted by $U(\mathfrak{g})$ and the center of $U(\mathfrak{g})$ is denoted by $Z(\mathfrak{g})$.

Fix a subset $\Theta \subset \Pi$. This defines a parabolic subalgebra $\mathfrak{p}_{\Theta} \supset \mathfrak{p}$. Let $\mathfrak{p}_{\Theta}=$ $\mathfrak{m}_{\Theta} \oplus \mathfrak{a}_{\Theta} \oplus \mathfrak{n}_{\Theta}$ be a Langlands decomposition such that $\mathfrak{a}_{\Theta} \subset \mathfrak{a}$ and $\mathfrak{n}_{\Theta} \supset \mathfrak{n}$. Denote the corresponding parabolic subgroup by $P_{\Theta, \mathbb{R}}=M_{\Theta, \mathbb{R}} A_{\Theta, \mathbb{R}} N_{\Theta, \mathbb{R}} \subset G_{\mathbb{R}}$. Let $\overline{\mathfrak{p}}_{\Theta}=\mathfrak{m}_{\Theta} \oplus \mathfrak{a}_{\Theta} \oplus \overline{\mathfrak{n}}_{\Theta}$ be a Langlands decomposition of the opposite subalgebra of $\mathfrak{p}_{\Theta}$. Put $\mathfrak{l}_{\Theta}=\mathfrak{m}_{\Theta} \oplus \mathfrak{a}_{\Theta}, L_{\Theta, \mathbb{R}}=M_{\Theta, \mathbb{R}} A_{\Theta, \mathbb{R}}$ and $K_{\Theta}=M_{\Theta} \cap K$.

Let $\mathcal{H C}_{\Theta}$ be the category of finitely generated $Z(\mathfrak{g})$-finite $\left(\mathfrak{g}, K_{\Theta} N_{\Theta}\right)$-modules. Here, a $\left(\mathfrak{g}, K_{\Theta} N_{\Theta}\right)$-module is a vector space equipped with a $\mathfrak{g}$-action and an algebraic $K_{\Theta} N_{\Theta}$-action satisfying the obvious compatibility. (We do not assume that the action of $K_{\Theta} N_{\Theta}$ is semisimple.) When we emphasis the group $G_{\mathbb{R}}$, we denote it by $\mathcal{H C}_{\Theta}^{G_{\mathbb{R}}}$. If $\Theta=\Pi$, then $\mathcal{H C}_{\Pi}$ is the category of Harish-Chandra modules (namely, finite length $(\mathfrak{g}, K)$-modules) of $G_{\mathbb{R}}$ [Wal88, 3.4.7. Corollary, 4.2.1. Theorem]. We will prove that $\mathcal{H C}_{\Theta}$ is equal to the category $\mathcal{O}_{P_{\Theta}, \mathbb{R}}^{\prime}$ defined by Hecht-Schmid [HS83b] (Lemma 3.1 and 3.2).

For $\mu \in \mathfrak{a}_{\Theta}^{*}$ and an $\mathfrak{a}_{\Theta}$-module $V$, denote the generalized $\mu$-weight space by $\Gamma_{\mu}(V)$. Set $\mathrm{wt}_{\mathfrak{a}_{\Theta}}(V)=\left\{\mu \in \mathfrak{a}_{\Theta}^{*} \mid \Gamma_{\mu}(V) \neq 0\right\}$.

Lemma 3.1. For a $\mathfrak{g}$-module $V$ and $k \in \mathbb{Z}_{>0}$, set $V_{k}=\left\{v \in V \mid \mathfrak{n}_{\Theta}^{k} v=0\right\}$.

(1) If $V_{1}$ is $Z\left(\mathfrak{l}_{\Theta}\right)$-finite, then $V_{k}$ is $Z\left(\mathfrak{l}_{\Theta}\right)$-finite for all $k \in \mathbb{Z}_{>0}$.

(2) If $V$ is $Z(\mathfrak{g})$-finite, then $V_{1}$ is $Z\left(\mathfrak{l}_{\Theta}\right)$-finite.

(3) If $V$ is a $Z(\mathfrak{g})$-finite $\left(\mathfrak{g}, N_{\Theta}\right)$-module, then $V=\bigoplus_{\mu \in \mathfrak{a}_{\Theta}^{*}} \Gamma_{\mu}(V)$.

Proof. (1) We prove (1) by induction on $k$. The homomorphism $V \rightarrow \operatorname{Hom}_{\mathbb{C}}\left(\mathfrak{n}_{\Theta}^{k}, V\right)$ defined by $v \mapsto(u \mapsto u v)$ gives a homomorphism $V_{k+1} / V_{k} \rightarrow \operatorname{Hom}_{\mathbb{C}}\left(\mathfrak{n}_{\Theta}^{k}, V_{1}\right)$, which is injective. By Kostant's theorem, $\operatorname{Hom}_{\mathbb{C}}\left(\mathfrak{n}_{\Theta}^{k}, V_{1}\right) \simeq\left(\mathfrak{n}_{\Theta}^{k}\right)^{*} \otimes V_{1}$ is $Z\left(\mathfrak{l}_{\Theta}\right)$-finite. Hence $V_{k+1}$ is $Z\left(\mathfrak{l}_{\Theta}\right)$-finite by inductive hypothesis.

(2) Consider the following homomorphism: $\varphi: Z(\mathfrak{g}) \hookrightarrow U(\mathfrak{g})=U\left(\mathfrak{l}_{\Theta}\right) \oplus\left(\overline{\mathfrak{n}}_{\Theta} U(\mathfrak{g})+\right.$ $\left.U(\mathfrak{g}) \mathfrak{n}_{\Theta}\right) \rightarrow U\left(\mathfrak{l}_{\Theta}\right)$. Then by a theorem of Harish-Chandra, the image of this homomorphism is contained in $Z\left(\mathfrak{l}_{\Theta}\right)$ and $Z\left(\mathfrak{l}_{\Theta}\right)$ is a finite $Z(\mathfrak{g})$-algebra. Moreover, the 
action of $Z(\mathfrak{g})$ on $V_{1}$ factors through $\varphi$. Set $I=\operatorname{Ann}_{Z(\mathfrak{g})} V$. Then $\varphi(I) Z\left(\mathfrak{l}_{\Theta}\right) V_{1}=0$ and $\varphi(I) Z\left(\mathfrak{l}_{\Theta}\right) \subset Z\left(\mathfrak{l}_{\Theta}\right)$ has a finite codimension.

(3) By (1) and (2), $V_{k}$ is $Z\left(\mathfrak{l}_{\Theta}\right)$-finite. Since $\mathfrak{a}_{\Theta}$ is contained in the center of $\mathfrak{l}_{\Theta}$, we have $U\left(\mathfrak{a}_{\Theta}\right) \subset Z\left(\mathfrak{l}_{\Theta}\right)$. Therefore, $V_{k}$ is $U\left(\mathfrak{a}_{\Theta}\right)$-finite. Hence we have $V_{k}=$ $\bigoplus_{\mu \in \mathfrak{a}_{\Theta}^{*}} \Gamma_{\mu}\left(V_{k}\right)$. By the assumption, we have $V=\bigcup_{k} V_{k}$. We get the lemma.

In particular, if $V \in \mathcal{H C}_{\Theta}$ then $V=\bigoplus_{\mu \in \mathfrak{a}_{\Theta}^{*}} \Gamma_{\mu}(V)$. Let $\Theta_{1}, \Theta_{2} \subset \Pi$ such that $\Theta_{2} \subset \Theta \subset \Theta_{1}$. For such data, we define $J_{\Theta_{2}, \Theta_{1}}(V)$ and $\widehat{J}_{\Theta_{2}, \Theta_{1}}(V)$ as follows:

$$
\begin{aligned}
& \widehat{J}_{\Theta_{2}, \Theta_{1}}(V)={\underset{k}{\leftarrow}}_{k} V /\left(\mathfrak{m}_{\Theta_{1}} \cap \overline{\mathfrak{n}}_{\Theta_{2}}\right)^{k} V, \\
& J_{\Theta_{2}, \Theta_{1}}(V)=\left\{v \in \widehat{J}_{\Theta_{2}, \Theta_{1}}(V) \mid \mathfrak{n}_{\Theta_{2}}^{k} v=0 \text { for some } k\right\} .
\end{aligned}
$$

If $\Theta_{1}=\Pi$ and $\Theta_{2}=\emptyset$, then $J_{\Theta_{2}, \Theta_{1}}(V)$ is called the Jacquet module of $V$. If $\Theta_{1}=\Theta_{2}=\Theta, J_{\Theta_{2}, \Theta_{1}}(V)=V$. We will prove the following properties in this section:

- For $V \in \mathcal{H C}_{\Theta}, J_{\Theta_{2}, \Theta_{1}}(V)$ is independent of $\Theta_{1}$ and an object of $\mathcal{H C}_{\Theta_{2}}$.

- For $V \in \mathcal{H C}_{\Theta_{1}}$ and $\Theta_{3} \subset \Theta_{2} \subset \Theta_{1} \subset \Pi$, we have $J_{\Theta_{3}, \Theta_{2}} \circ J_{\Theta_{2}, \Theta_{1}}(V) \simeq$ $J_{\Theta_{3}, \Theta_{1}}(V)$.

- The functor $J_{\Theta_{2}, \Theta_{1}}: \mathcal{H C}_{\Theta_{1}} \rightarrow \mathcal{H C}_{\Theta_{2}}$ is exact.

So the usual Jacquet functor is decomposed into the composite of $J_{\Theta_{2}, \Theta_{1}}$ 's.

Lemma 3.2. (1) If $V \in \mathcal{H C}_{\Theta}^{G_{\mathbb{R}}}$ then $\Gamma_{\mu_{1}}(V) \in \mathcal{H C}_{\Theta}^{L_{\Theta_{1}, \mathbb{R}}}$ for $\mu_{1} \in \mathfrak{a}_{\Theta_{1}}^{*}$.

(2) If $V \in \mathcal{H C}_{\Theta}^{G_{\mathbb{R}}}$ then $V / \overline{\mathfrak{n}}_{\Theta} V \in \mathcal{H C}_{\Theta}^{L_{\Theta, \mathbb{R}}}$.

(3) If $V \in \mathcal{H C}_{\Pi}^{G_{\mathbb{R}}}$ then $V / \overline{\mathfrak{n}}_{\Theta} V \in \mathcal{H C}_{\Theta}^{L_{\Theta, \mathbb{R}}}$.

(4) For $V \in \mathcal{H C}_{\Theta}$ and $\mu_{1} \in \mathfrak{a}_{\Theta_{1}}^{*}, \Gamma_{\mu_{1}}\left(V /\left(\mathfrak{m}_{\Theta_{1}} \cap \overline{\mathfrak{n}}_{\Theta_{2}}\right)^{k} V\right)$ is a Harish-Chandra module of $L_{\Theta_{2}, \mathbb{R}}$.

Proof. (1) It is easy to see that $\Gamma_{\mu_{1}}(V)$ is a $\left(\mathfrak{g}, K_{\Theta}\left(M_{\Theta_{1}} \cap N_{\Theta}\right)\right)$-module. It is sufficient to prove that $\Gamma_{\mu_{1}}(V)$ is a finitely generated $U\left(\mathfrak{l}_{\Theta_{1}}\right)$-module and $Z\left(\mathfrak{l}_{\Theta_{1}}\right)$ finite.

Since $V \in \mathcal{H C}_{\Theta}, V$ is generated by a finite-dimensional subspace $W$ of $V$. By Lemma 3.1 (3), the action of $\mathfrak{a}_{\Theta}$ on $V$ is locally finite. By the definition of $\mathcal{H C} \mathcal{C}_{\Theta}$, the action of $\mathfrak{n}_{\Theta}$ on $V$ is locally finite. Hence we may assume that $W$ is $\mathfrak{n}_{\Theta} \oplus \mathfrak{a}_{\Theta}$-stable. In particular, $W$ is $\mathfrak{n}_{\Theta_{1}} \oplus \mathfrak{a}_{\Theta_{1}}$-stable. Therefore, we have $V=U\left(\mathfrak{m}_{\Theta_{1}}\right) U\left(\overline{\mathfrak{n}}_{\Theta_{1}}\right) W$. From this, we get

$$
\Gamma_{\mu_{1}}(V)=U\left(\mathfrak{m}_{\Theta_{1}}\right)\left(\sum_{\mu_{1}^{\prime} \in \mathrm{wt}_{\mathfrak{a}_{\Theta_{1}}}(W)} \Gamma_{\mu_{1}-\mu_{1}^{\prime}}\left(U\left(\overline{\mathfrak{n}}_{\Theta_{1}}\right)\right) \Gamma_{\mu_{1}^{\prime}}(W)\right) .
$$

Since $W$ is finite-dimensional, $\mathrm{wt}_{\mathfrak{a}_{\Theta_{1}}}(W)$ is finite. For each $\mu_{1}^{\prime}, \Gamma_{\mu_{1}-\mu_{1}^{\prime}}\left(U\left(\overline{\mathfrak{n}}_{\Theta_{1}}\right)\right)$ is finite-dimensional. Therefore, $\sum_{\mu_{1}^{\prime} \in \mathrm{wt}_{\mathrm{a}_{\Theta_{1}}}(W)} \Gamma_{\mu_{1}-\mu_{1}^{\prime}}\left(U\left(\overline{\mathfrak{n}}_{\Theta_{1}}\right)\right) \Gamma_{\mu_{1}^{\prime}}(W)$ is finitedimensional. Hence $\Gamma_{\mu_{1}}(V)$ is a finitely generated $U\left(\mathfrak{m}_{\Theta_{1}}\right)$-module.

Put $V_{k}=\left\{v \in V \mid \mathfrak{n}_{\Theta_{1}}^{k} v=0\right\}$. Since $\Gamma_{\mu_{1}}(V)$ is finitely generated $U\left(\mathfrak{m}_{\Theta_{1}}\right)$ module, we can take $\mathfrak{n}_{\Theta_{1}}$-stable subspace $W^{\prime}$ such that $\Gamma_{\mu_{1}}(V) \subset U\left(\mathfrak{m}_{\Theta_{1}}\right) W^{\prime}$. Since $W^{\prime}$ is finite-dimensional, $W^{\prime} \subset V_{k}$ for some $k \in \mathbb{Z}_{\geq 0}$. By Lemma 3.1] $V_{k}$ is $Z\left(\mathfrak{l}_{\Theta_{1}}\right)$ finite. Hence $\Gamma_{\mu_{1}}(V)$ is $Z\left(\mathfrak{l}_{\Theta_{1}}\right)$-finite.

(2) As above, we can take a finite-dimensional $\mathfrak{n}_{\Theta} \oplus \mathfrak{a}_{\Theta}$-stable submodule $W$ which generates $V$ as a $\mathfrak{g}$-module. Then we have $V=U\left(\overline{\mathfrak{n}}_{\Theta}\right) U\left(\mathfrak{m}_{\Theta}\right) W$. Hence we 
get a surjective homomorphism $U\left(\mathfrak{m}_{\Theta}\right) W \rightarrow V / \overline{\mathfrak{n}}_{\Theta} V$. It is sufficient to prove that $U\left(\mathfrak{m}_{\Theta}\right) W \in \mathcal{H C}_{\Theta}^{L_{\Theta, \mathbb{R}}}$. Since $W$ is finite-dimensional, $W \subset \bigoplus_{\mu \in \Lambda} \Gamma_{\mu}(V)$ for a finite subset $\Lambda \subset \mathfrak{a}_{\Theta}^{*}$. Each $\Gamma_{\mu}(V)$ is $\mathfrak{m}_{\Theta}$-stable. Therefore, $U\left(\mathfrak{m}_{\Theta}\right) W \subset \bigoplus_{\mu \in \Lambda} \Gamma_{\mu}(V)$. By $(1), \Gamma_{\mu}(V) \in \mathcal{H C}_{\Theta}^{L_{\Theta, \mathbb{R}}}$. Hence we have $U\left(\mathfrak{m}_{\Theta}\right) W \in \mathcal{H C}_{\Theta}^{L_{\Theta, \mathbb{R}}}$.

(3) Recall that an object of $\mathcal{H C}_{\Pi}^{G_{\mathbb{R}}}$ is a Harish-Chandra module of $G_{\mathbb{R}}$. Hence this is [HS83a, Proposition 2.24].

(4) By Lemma 3.1, we have $V=\bigoplus_{\mu_{1} \in \mathfrak{a}_{\Theta}^{*}} \Gamma_{\mu_{1}}(V)$. Since $\mathfrak{m}_{\Theta_{1}}$ has an $\mathfrak{a}_{\Theta_{1}}$-weight 0 , the action of $\mathfrak{m}_{\Theta}$ preserves each $\Gamma_{\mu_{1}}(V)$. Therefore, we have $\Gamma_{\mu_{1}}\left(V /\left(\mathfrak{m}_{\Theta_{1}} \cap\right.\right.$ $\left.\left.\overline{\mathfrak{n}}_{\Theta_{2}}\right)^{k} V\right)=\Gamma_{\mu_{1}}(V) /\left(\mathfrak{m}_{\Theta_{1}} \cap \overline{\mathfrak{n}}_{\Theta_{2}}\right)^{k} \Gamma_{\mu_{1}}(V)$. By $(1), \Gamma_{\mu_{1}}(V) \in \mathcal{H C}_{\Theta}^{L_{\Theta_{1}, \mathbb{R}}}$. Hence we may assume that $\Theta_{1}=\Pi$. So we have $\mathfrak{m}_{\Theta_{1}} \cap \overline{\mathfrak{n}}_{\Theta_{2}}=\overline{\mathfrak{n}}_{\Theta_{2}}$.

By $(2), V^{\prime}=V / \overline{\mathfrak{n}}_{\Theta} V \in \mathcal{H C}_{\Theta}^{L_{\Theta, \mathbb{R}}}$. By $(3), V^{\prime \prime}=V^{\prime} /\left(\mathfrak{m}_{\Theta} \cap \overline{\mathfrak{n}}_{\Theta_{2}}\right) V^{\prime} \in \mathcal{H C}_{\Theta_{2}}^{L_{\Theta_{2}, \mathbb{R}}}$. Since $V^{\prime \prime}=V / \overline{\mathfrak{n}}_{\Theta_{2}} V$, we get the lemma for $k=1$.

For a general $k$, we can prove the lemma by induction on $k$ using an exact sequence $\overline{\mathfrak{n}}_{\Theta_{2}}^{k} \otimes\left(V / \overline{\mathfrak{n}}_{\Theta_{2}} V\right) \rightarrow V / \overline{\mathfrak{n}}_{\Theta_{2}}^{k+1} V \rightarrow V / \overline{\mathfrak{n}}_{\Theta_{2}}^{k} V \rightarrow 0$.

We now prove that the length of an object of $\mathcal{H C}_{\Theta}$ is finite.

Lemma 3.3. Assume that a $\left(\mathfrak{g}, N_{\Theta}\right)$-module $V$ satisfies the following conditions:

(1) The module $V$ is $Z(\mathfrak{g})$-finite.

(2) For all $\mu \in \mathfrak{a}_{\Theta}^{*}, \Gamma_{\mu}(V)$ has a finite length as a $\mathfrak{m}_{\Theta}$-module.

Then $V$ has a finite length.

Proof. Let $\varphi: Z(\mathfrak{g}) \rightarrow Z\left(\mathfrak{l}_{\Theta}\right)$ be a homomorphism defined in the proof of Lemma 3.1. Set $J=\varphi\left(\operatorname{Ann}_{Z(\mathfrak{g})} V\right) Z\left(\mathfrak{l}_{\Theta}\right)$. Then $J$ has a finite codimension. In particular, there exists a finite subset $\Lambda \subset \mathfrak{a}_{\Theta}^{*}$ which gives all maximal ideals of $U\left(\mathfrak{a}_{\Theta}\right) /(J \cap$ $\left.U\left(\mathfrak{a}_{\Theta}\right)\right)$. Let $V^{\prime}$ be a subquotient of $V$. Then we have $J\left(V^{\prime}\right)^{\mathfrak{n}_{\Theta}}=0$, hence $\left(V^{\prime}\right)^{\mathfrak{n}_{\Theta}} \subset$ $\bigoplus_{\mu \in \Lambda} \Gamma_{\mu}\left(V^{\prime}\right)$. Since $V^{\prime}$ is a $\left(\mathfrak{g}, N_{\Theta}\right)$-module, the space $\left(V^{\prime}\right)^{\mathfrak{n}_{\Theta}}$ is non-zero. Hence the length of the $\mathfrak{g}$-module $V$ is less than or equal to the sum of the length of $\mathfrak{m}_{\Theta}$-modules $\Gamma_{\mu}(V)$ for $\mu \in \Lambda$. It is finite by the assumption.

Corollary 3.4. Each object in $\mathcal{H C}_{\Theta}$ has a finite length.

Proof. The condition of (1) in the previous lemma is satisfied by the definition of $\mathcal{H C}_{\Theta}$. For $V \in \mathcal{H C}_{\Theta}$ and $\mu \in \mathfrak{a}_{\Theta}^{*}, \Gamma_{\mu}(V)$ is a Harish-Chandra module of $L_{\Theta, \mathbb{R}}$ by Lemma 3.2 (4). (Take $\Theta_{1}=\Theta_{2}=\Theta$.) Hence it has a finite length. By the previous lemma, we get the corollary.

For a subset $\Lambda \subset \mathfrak{a}_{\Theta}^{*}$, put $\Lambda-\left.\mathbb{Z}_{\geq 0} \Pi\right|_{\mathfrak{a}_{\Theta}}=\left\{\mu-\left.\sum_{\alpha \in \Pi} n_{\alpha} \alpha\right|_{\mathfrak{a}_{\Theta}} \mid \mu \in \Lambda, n_{\alpha} \in \mathbb{Z}_{\geq 0}\right\}$.

Lemma 3.5. For $V \in \mathcal{H C}_{\Theta}$, there exists a finite subset $\Lambda_{2}$ of $\mathfrak{a}_{\Theta_{2}}^{*}$ such that $\mathrm{wt}_{\mathfrak{a}_{\Theta_{2}}}\left(\widehat{J}_{\Theta_{2}, \Theta_{1}}(V)\right) \subset \Lambda_{2}-\left.\mathbb{Z}_{\geq 0} \Pi\right|_{\mathfrak{a}_{\Theta_{2}}}$.

Proof. Put $\mathfrak{c}=\mathfrak{m}_{\Theta_{1}} \cap \overline{\mathfrak{n}}_{\Theta_{2}}$. As in the proof of Lemma 3.2, we can take a finitedimensional $\mathfrak{n}_{\Theta_{1}} \oplus \mathfrak{a}_{\Theta_{1}}$-stable subspace $W$ such that $V=U(\mathfrak{g}) W$. Put $\Lambda_{1}=$ $\mathrm{wt}_{\mathfrak{a}_{\Theta_{1}}}(W)$. Then this is finite and, since $V=U\left(\mathfrak{m}_{\Theta}\right) U\left(\overline{\mathfrak{n}}_{\Theta}\right) W$, wt $\mathfrak{a}_{\mathfrak{a}_{1}}(V) \subset \Lambda_{1}-$ $\left.\mathbb{Z}_{\geq 0} \Pi\right|_{\mathfrak{a}_{\Theta_{1}}}$. Set $\Lambda_{2}=\bigcup_{\mu_{1} \in \Lambda_{1}} \mathrm{wt}_{\mathfrak{a}_{\Theta_{2}}}\left(\Gamma_{\mu_{1}}(V / \mathfrak{c} V)\right)=\left\{\mu_{2} \in \mathrm{wt}_{\mathfrak{a}_{\Theta_{2}}}(V / \mathfrak{c} V)\left|\mu_{2}\right|_{\mathfrak{a}_{\Theta_{1}}} \in\right.$

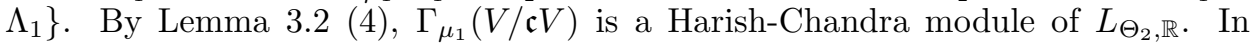
particular, it is $Z\left(\mathfrak{l}_{\Theta}\right)$-finite. Therefore, it is $U\left(\mathfrak{a}_{\Theta}\right)$-finite since $\mathfrak{a}_{\Theta}$ is a subalgebra of the center of $\mathfrak{l}_{\Theta}$. Hence $\mathrm{wt}_{\mathfrak{a}_{\Theta_{2}}}\left(\Gamma_{\mu_{1}}(V / \mathfrak{c} V)\right)$ is finite. This implies that $\Lambda_{2}$ is finite. We also have $\mathrm{wt}_{\mathfrak{a}_{\Theta_{2}}}(V / \mathfrak{c} V) \subset \Lambda_{2}-\left.\mathbb{Z}_{\geq 0} \Pi\right|_{\mathfrak{a}_{\Theta_{2}}}$. 
We prove $\mathrm{wt}_{\mathfrak{a}_{\Theta_{2}}}\left(V / \mathfrak{c}^{k} V\right) \subset \Lambda_{2}-\left.\mathbb{Z}_{\geq 0} \Pi\right|_{\mathfrak{a}_{\Theta_{2}}}$ by induction on $k$. Then we get the lemma. From an exact sequence $\mathfrak{c} \otimes\left(V / \mathfrak{c}^{k} V\right) \rightarrow V / \mathfrak{c}^{k+1} V \rightarrow V / \mathfrak{c} V \rightarrow 0$, we have

$$
\operatorname{wt}\left(V / \mathfrak{c}^{k+1} V\right) \subset \operatorname{wt}\left(\mathfrak{c} \otimes\left(V / \mathfrak{c}^{k} V\right)\right) \cup \operatorname{wt}(V / \mathfrak{c} V) \subset \Lambda_{2}-\left.\mathbb{Z}_{\geq 0} \Pi\right|_{\mathfrak{a}_{2}}
$$

by the inductive hypothesis.

Lemma 3.6. For $V \in \mathcal{H C}_{\Theta}$, we have $J_{\Theta_{2}, \Theta_{1}}(V)=\bigoplus_{\mu_{2} \in \mathfrak{a}_{\Theta_{2}}^{*}} \Gamma_{\mu_{2}}\left(\widehat{J}_{\Theta_{2}, \Theta_{1}}(V)\right)$. Therefore, $\Gamma_{\mu_{2}}\left(\widehat{J}_{\Theta_{2}, \Theta_{1}}(V)\right)=\Gamma_{\mu_{2}}\left(J_{\Theta_{2}, \Theta_{1}}(V)\right)$.

Proof. By Lemma 3.5, the right hand side is contained in the left hand side. On the other hand, by Lemma 3.1 (3), we have $J_{\Theta_{2}, \Theta_{1}}(V)=\bigoplus_{\mu_{2} \in \mathfrak{a}_{\Theta_{2}}^{*}} \Gamma_{\mu_{2}}\left(J_{\Theta_{2}, \Theta_{1}}(V)\right)$. This is a subspace of the right hand side.

Notice that $V /\left(\mathfrak{m}_{\Theta_{1}} \cap \overline{\mathfrak{n}}_{\Theta_{2}}\right)^{k} V \simeq \widehat{J}_{\Theta_{2}, \Theta_{1}}(V) /\left(\mathfrak{m}_{\Theta_{1}} \cap \overline{\mathfrak{n}}_{\Theta_{2}}\right)^{k} \widehat{J}_{\Theta_{2}, \Theta_{1}}(V)$ by the definition.

Lemma 3.7. For $\mu_{2} \in \mathfrak{a}_{\Theta_{2}}^{*}$, there exists $k \in \mathbb{Z}_{\geq 0}$ such that $\Gamma_{\mu_{2}}\left(\widehat{J}_{\Theta_{2}, \Theta_{1}}(V)\right) \rightarrow$ $\Gamma_{\mu_{2}}\left(V /\left(\mathfrak{m}_{\Theta_{1}} \cap \overline{\mathfrak{n}}_{\Theta_{2}}\right)^{k} V\right)$ is an isomorphism.

Proof. Put $\mathfrak{c}=\mathfrak{m}_{\Theta_{1}} \cap \overline{\mathfrak{n}}_{\Theta_{2}}$. Take $\Lambda_{2}$ as in Lemma 3.5. Let $k \in \mathbb{Z}_{\geq 0}$ such that for any $\alpha_{1}, \ldots, \alpha_{k} \in \Sigma^{+}$we have $\mu_{2} \notin\left(\Lambda_{2}-\left.\mathbb{Z}_{\geq 0} \Pi\right|_{\mathfrak{a}_{\Theta_{2}}}\right)-\left(\alpha_{1}+\cdots+\alpha_{k}\right)$. Then we have $\Gamma_{\mu_{2}}\left(\mathfrak{c}^{k} \widehat{J}_{\Theta_{2}, \Theta_{1}}(V)\right)=0$. By the exact sequence $0 \rightarrow \mathfrak{c}^{k} \widehat{J}_{\Theta_{2}, \Theta_{1}}(V) \rightarrow \widehat{J}_{\Theta_{2}, \Theta_{1}}(V) \rightarrow$ $V / \mathfrak{c}^{k} V \rightarrow 0$, we have $0=\Gamma_{\mu_{2}}\left(\mathfrak{c}^{k}{\widehat{J_{\Theta}}, \Theta_{1}}(V)\right) \rightarrow \Gamma_{\mu_{2}}\left(\widehat{J}_{\Theta_{2}, \Theta_{1}}(V)\right) \rightarrow \Gamma_{\mu_{2}}\left(V / \mathfrak{c}^{k} V\right) \rightarrow 0$. Hence we get the lemma.

Lemma 3.8. For $V \in \mathcal{H C} \mathcal{C}_{\Theta}$ and $\mu_{2} \in \mathfrak{a}_{\Theta_{2}}^{*}, \Gamma_{\mu_{2}}\left(J_{\Theta_{2}, \Theta_{1}}(V)\right)$ is a Harish-Chandra module for $L_{\Theta_{2}, \mathbb{R}}$.

Proof. This follows from Lemma 3.2 Lemma 3.6 and Lemma 3.7

If $\Theta=\Theta_{1}=\Pi$, the following proposition is HS83b, (34) Lemma].

Proposition 3.9. If $V \in \mathcal{H C}_{\Theta}$, then $J_{\Theta_{2}, \Theta_{1}}(V) \in \mathcal{H C}_{\Theta_{2}}$.

Proof. It is sufficient to prove that $J_{\Theta_{2}, \Theta_{1}}(V)$ has a finite length. This follows from Lemma 3.3 and the previous lemma.

Hence $J_{\Theta_{2}, \Theta_{1}}$ defines a functor $\mathcal{H C}_{\Theta} \rightarrow \mathcal{H C}_{\Theta_{2}}$.

Proposition 3.10. For $\Theta_{3} \subset \Theta_{2} \subset \Theta \subset \Theta_{1} \subset \Pi$, we have $J_{\Theta_{3}, \Theta_{2}} \circ J_{\Theta_{2}, \Theta_{1}} \simeq$ $J_{\Theta_{3}, \Theta_{1}}: \mathcal{H C}_{\Theta} \rightarrow \mathcal{H C}_{\Theta_{3}}$.

We use the following lemma.

Lemma 3.11. Let $\mathfrak{c}$ be a finite-dimensional nilpotent Lie algebra, $\mathfrak{c}_{1}, \mathfrak{c}_{2} \subset \mathfrak{c}$ Lie subalgebras such that:

- $\mathfrak{c}_{2}$ is an ideal of $\mathfrak{c}$.

- $\mathfrak{c}=\mathfrak{c}_{1} \oplus \mathfrak{c}_{2}$.

Then for all $k_{1}, k_{2} \in \mathbb{Z}_{\geq 0}$ there exists $n \in \mathbb{Z}_{\geq 0}$ such that $\mathfrak{c}^{n} \subset \mathfrak{c}_{1}^{k_{1}} U(\mathfrak{c})+\mathfrak{c}_{2}^{k_{2}} U(\mathfrak{c})$.

Proof. Set $V=U(\mathfrak{c}) /\left(\mathfrak{c}_{1}^{k_{1}} U(\mathfrak{c})+\mathfrak{c}_{2}^{k_{2}} U(\mathfrak{c})\right), v_{0}=1 \in V$. Then $V$ is a right $U(\mathfrak{c})$ module, $V=v_{0} U(\mathfrak{c})$ and $v_{0} \mathfrak{c}_{1}^{k_{1}}=v_{0} \mathfrak{c}_{2}^{k_{2}}=0$. We have $V=v_{0} U\left(\mathfrak{c}_{1}\right) U\left(\mathfrak{c}_{2}\right)$. Since $v_{0} \mathfrak{c}_{1}^{k_{1}}=0, v_{0} U\left(\mathfrak{c}_{1}\right)$ is finite-dimensional. By the assumption, $\mathfrak{c}_{2}$ is an ideal of c. Therefore, $v_{0} U\left(\mathfrak{c}_{1}\right) \mathfrak{c}_{2}^{k_{2}}=v_{0} \mathfrak{c}_{2}^{k_{2}} U(\mathfrak{c})=0$. Hence $V$ is finite-dimensional. Since 
a finite-dimensional irreducible representation of $\mathfrak{c}$ is a character, $V$ is given by an extension of characters. As $v_{0} \mathfrak{c}_{1}^{k_{1}}=v_{0} \mathfrak{c}_{2}^{k_{2}}=0$ and $\mathfrak{c}$ is nilpotent, for every $v \in V$ there exist integers $l_{1}, l_{2}$ such that $v \mathfrak{c}_{1}^{l_{1}}=v \mathfrak{c}_{2}^{l_{2}}=0$. This implies that each irreducible subquotient of $V$ is the trivial representation. Hence there exists $n$ such that $v_{0} \mathfrak{c}^{n}=0$. This completes the proof.

Proof of Proposition 3.10. We prove that for each $\mu_{3} \in \mathfrak{a}_{\Theta_{3}}^{*}$ the generalized $\mu_{3^{-}}$ weight spaces of both sides are isomorphic. Put $\mathfrak{c}_{1}=\mathfrak{m}_{\Theta_{2}} \cap \overline{\mathfrak{n}}_{\Theta_{3}}, \mathfrak{c}_{2}=\mathfrak{m}_{\Theta_{1}} \cap \overline{\mathfrak{n}}_{\Theta_{2}}$ and $\mathfrak{c}=\mathfrak{c}_{1} \oplus \mathfrak{c}_{2}$. Then $\mathfrak{c}_{1}, \mathfrak{c}_{2}$ satisfies the assumption of the previous lemma and $\mathfrak{c}=\mathfrak{m}_{\Theta_{1}} \cap \overline{\mathfrak{n}}_{\Theta_{3}}$. Put $\mu_{2}=\left.\mu_{3}\right|_{\mathfrak{a}_{\Theta_{2}}}$. By Lemma 3.6 and Lemma 3.7, for sufficiently large $k_{1}, k_{2}$, we have

$$
\begin{aligned}
\Gamma_{\mu_{3}}\left(J_{\Theta_{3}, \Theta_{2}}\left(J_{\Theta_{2}, \Theta_{1}}(V)\right)\right) & =\Gamma_{\mu_{3}}\left(J_{\Theta_{2}, \Theta_{1}}(V) / \mathfrak{c}_{1}^{k_{1}} J_{\Theta_{2}, \Theta_{1}}(V)\right) \\
& =\Gamma_{\mu_{3}} \Gamma_{\mu_{2}}\left(J_{\Theta_{2}, \Theta_{1}}(V) / \mathfrak{c}_{1}^{k_{1}} J_{\Theta_{2}, \Theta_{1}}(V)\right) \\
& =\Gamma_{\mu_{3}}\left(\Gamma_{\mu_{2}}\left(J_{\Theta_{2}, \Theta_{1}}(V)\right) / \mathfrak{c}_{1}^{k_{1}} \Gamma_{\mu_{2}}\left(J_{\Theta_{2}, \Theta_{1}}(V)\right)\right) \\
& =\Gamma_{\mu_{3}}\left(\Gamma_{\mu_{2}}\left(V / \mathfrak{c}_{2}^{k_{2}} V\right) / \mathfrak{c}_{1}^{k_{1}} \Gamma_{\mu_{2}}\left(V / \mathfrak{c}_{2}^{k_{2}} V\right)\right) \\
& =\Gamma_{\mu_{3}}\left(\Gamma_{\mu_{2}}\left(\left(V / \mathfrak{c}_{2}^{k_{2}} V\right) / \mathfrak{c}_{1}^{k_{1}}\left(V / \mathfrak{c}_{2}^{k_{2}} V\right)\right)\right) \\
& =\Gamma_{\mu_{3}}\left(\Gamma_{\mu_{2}}\left(V /\left(\mathfrak{c}_{1}^{k_{1}} V+\mathfrak{c}_{2}^{k_{2}} V\right)\right)\right) \\
& =\Gamma_{\mu_{3}}\left(V /\left(\mathfrak{c}_{1}^{k_{1}} V+\mathfrak{c}_{2}^{k_{2}} V\right)\right)
\end{aligned}
$$

We also have

$$
\Gamma_{\mu_{3}}\left(J_{\Theta_{3}, \Theta_{1}}(V)\right) \simeq \Gamma_{\mu_{3}}\left(V / \mathfrak{c}^{k} V\right)
$$

for sufficiently large $k$. Fix $k_{1}, k_{2}$ and take $n$ as in the previous lemma. We may assume $k \leq k_{1}, k_{2} \leq n$. Consider the following homomorphism:

$$
\Gamma_{\mu_{3}}\left(V / \mathfrak{c}^{n} V\right) \rightarrow \Gamma_{\mu_{3}}\left(V /\left(\mathfrak{c}_{1}^{k_{1}} V+\mathfrak{c}_{2}^{k_{2}} V\right)\right) \rightarrow \Gamma_{\mu_{3}}\left(V / \mathfrak{c}^{k} V\right) .
$$

Since $V / \mathfrak{c}^{n} V$ is decomposed into the generalized $\mathfrak{a}_{\Theta_{3}}$-weight spaces (this follows from the fact that $V / \mathfrak{c}^{n} V$ is a Harish-Chandra module of $\left.L_{\Theta_{3}, \mathbb{R}}\right)$, the first homomorphism is surjective. If $k_{1}, k_{2}, n, k$ is sufficiently large, the composition of this homomorphism is isomorphic by Lemma 3.7. Hence the first homomorphism is injective. We get the proposition.

Proposition 3.12. The functor $J_{\Theta_{2}, \Theta_{1}}: \mathcal{H C}_{\Theta} \rightarrow \mathcal{H C}_{\Theta_{2}}$ is independent of $\Theta_{1}$.

Proof. By the previous proposition, $J_{\Theta_{2}, \Theta_{1}}=J_{\Theta_{2}, \Theta} \circ J_{\Theta, \Theta_{1}}$. Hence it is sufficient to prove that $J_{\Theta, \Theta_{1}}(V) \simeq V$ for $V \in \mathcal{H C}_{\Theta}$. We compare the $\mu$-weight spaces for each $\mu \in \mathfrak{a}_{\Theta}^{*}$.

Put $\mathfrak{c}=\mathfrak{m}_{\Theta_{1}} \cap \overline{\mathfrak{n}}_{\Theta}$. Take a finite subset $\Lambda \subset \mathfrak{a}_{\Theta}^{*}$ such that $\operatorname{wt}_{\mathfrak{a}_{\Theta}}(V) \subset \Lambda-$ $\left.\mathbb{Z}_{\geq 0} \Pi\right|_{\mathfrak{a}_{\Theta}}$. Then for a sufficiently large $k$, for any $\alpha_{1}, \ldots, \alpha_{k} \in \Pi$ we have $\mu \notin$ $\Lambda-\left.\mathbb{Z}_{\geq 0} \Pi\right|_{\mathfrak{a}_{\Theta}}-\left(\alpha_{1}+\cdots+\alpha_{k}\right)$. Hence we have $\mu \notin \mathrm{wt}_{\mathfrak{a}_{\Theta}}\left(\mathfrak{c}^{k} V\right)$. This implies $\Gamma_{\mu}(V) \simeq \Gamma_{\mu}\left(V / \mathfrak{c}^{k} V\right)$. On the other hand, the right hand side is isomorphic to $\Gamma_{\mu}\left(J_{\Theta, \Theta_{1}}(V)\right)$ for a sufficiently large $k$ by Lemma 3.7 We get the proposition.

Lemma 3.13. Each $V \in \mathcal{H C}_{\Theta}$ is finitely generated as a $U(\overline{\mathfrak{n}})$-module.

Proof. Take a finite-dimensional $\mathfrak{n}_{\Theta} \oplus \mathfrak{a}_{\Theta}$-stable subspace $W$ of $V$ which generates $V$ as a $\mathfrak{g}$-module. Then $U\left(\mathfrak{m}_{\Theta}\right) W \subset \bigoplus_{\mu \in \Lambda} \Gamma_{\mu}(V)$ for some finite subset $\Lambda \subset \mathfrak{a}_{\Theta}^{*}$. Hence $U\left(\mathfrak{m}_{\Theta}\right) W$ is a Harish-Chandra module of $L_{\Theta, \mathbb{R}}$. Therefore, by a theorem of Casselman-Osborne [CO78, 2.3 Theorem], $U\left(\mathfrak{m}_{\Theta}\right) W$ is finitely generated as a 
$\left(\mathfrak{m}_{\Theta} \cap \overline{\mathfrak{n}}\right)$-module. Since $V=U\left(\overline{\mathfrak{n}}_{\Theta}\right) U\left(\mathfrak{m}_{\Theta}\right) W, V$ is a finitely generated $U\left(\overline{\mathfrak{n}}_{\Theta}\right) U\left(\mathfrak{m}_{\Theta} \cap\right.$ $\overline{\mathfrak{n}})=U(\overline{\mathfrak{n}})$-module.

Proposition 3.14. The functor $J_{\Theta_{2}, \Theta_{1}}: \mathcal{H C}_{\Theta_{1}} \rightarrow \mathcal{H C}_{\Theta_{2}}$ is exact.

Proof. If $\Theta_{2}=\emptyset$ and $\Theta_{1}=\Pi$, this proposition is well-known Wal88, 4.1.5. Theorem]. The key point of the proof is the Artin-Rees property and that $V \in \mathcal{H C}_{\Pi}$ is finitely generated as a $U(\overline{\mathfrak{n}})$-module. Hence the usual proof is applicable for our situation using the above lemma.

\section{The Geometric JacQuet FunCtor}

In this section, we recall an argument of Emerton-Nadler-Vilonen [ENV04. For $\Theta \subset \Pi$, let $\mathcal{H C}_{\Theta, \rho}$ be the category of $V \in \mathcal{H C}_{\Theta}$ whose infinitesimal character is the same as that of the trivial representation. Fix a Borel subgroup $B$ of $G$. Then by the Beilinson-Bernstein correspondence and the Riemann-Hilbert correspondence, we have an equivalence of categories $\Delta: \mathcal{H C}_{\Theta, \rho} \simeq \operatorname{Perv}_{K_{\Theta} N_{\Theta}}(G / B)$ where $\operatorname{Perv}_{K_{\Theta} N_{\Theta}}(G / B)$ is the category of $K_{\Theta} N_{\Theta}$-equivariant perverse sheaves on $G / B$.

Fix a cocharacter $\nu: \mathbb{G}_{m} \rightarrow A$ such that $\langle\nu, \alpha\rangle \geq 0$ for all $\alpha \in \Pi$. Define $a_{\nu}: G / B \times \mathbb{G}_{m} \rightarrow G / B$ by $(g B, t) \mapsto \nu(t) g B$. Let $R \psi$ be the nearby cycle functor with respect to $G / B \times \mathbb{A}^{1} \rightarrow \mathbb{A}^{1}$. For $\mathscr{F} \in \operatorname{Perv}(G / B)$, put $\Psi_{\nu}(\mathscr{F})=R \psi a_{\nu}^{*} \mathscr{F}$. Then the main theorem of ENV04 is the following.

Theorem 4.1 (Emerton-Nadler-Vilonen ENV04, Theorem 1.1]). Assume that $\nu$ is regular. We have $\Delta \circ J_{\emptyset, \Pi} \simeq \Psi_{\nu} \circ \Delta: \mathcal{H C}_{\Pi, \rho} \rightarrow \operatorname{Perv}(G / B)$.

Their argument can be applicable for a general $\nu$. Namely, we can prove the following theorem.

Theorem 4.2. Set $\Theta=\{\alpha \in \Pi \mid\langle\alpha, \nu\rangle=0\}$. Then we have $\Delta \circ J_{\Theta, \Theta^{\prime}} \simeq$ $\Psi_{\nu} \circ \Delta: \mathcal{H C}_{\Theta^{\prime}, \rho} \rightarrow \operatorname{Perv}(G / B)$ for all $\Theta^{\prime} \subset \Pi$ such that $\Theta \subset \Theta^{\prime}$.

We review the proof. Let $V \in \mathcal{H C}_{\Theta^{\prime}, \rho}$. First we construct a filtration on $V$. To construct it, we prove the following lemma.

Lemma 4.3. We have the following.

(1) We have $J_{\Theta, \Pi}(V) / \overline{\mathfrak{n}}_{\Theta}^{k} J_{\Theta, \Pi}(V) \simeq \widehat{J}_{\Theta, \Pi}(V) / \overline{\mathfrak{n}}_{\Theta}^{k} \widehat{J}_{\Theta, \Pi}(V)$.

(2) We have $\widehat{J}_{\Theta, \Pi}(V) \simeq \widehat{J}_{\Theta, \Pi}\left(J_{\Theta, \Pi}(V)\right)$.

(3) We have $\widehat{J}_{\Theta, \Pi}(V) \simeq \prod_{\mu \in \mathfrak{a}_{\Theta}^{*}} \Gamma_{\mu}\left(J_{\Theta, \Pi}(V)\right)$.

(4) The homomorphism $V \rightarrow \widehat{J}_{\Theta, \Pi}(V)$ is injective.

Proof. (1) Since both sides are decomposed into generalized $\mathfrak{a}_{\Theta}^{*}$-weight spaces, it is sufficient to prove that the $\mu$-weight spaces of both sides are isomorphic for every $\mu \in \mathfrak{a}_{\Theta}^{*}$. We have

$$
\Gamma_{\mu}\left(J_{\Theta, \Pi}(V) / \overline{\mathfrak{n}}_{\Theta}^{k} J_{\Theta, \Pi}(V)\right) \simeq \Gamma_{\mu}\left(J_{\Theta, \Pi}(V)\right) / \Gamma_{\mu}\left(\overline{\mathfrak{n}}_{\Theta}^{k} J_{\Theta, \Pi}(V)\right) .
$$

By Lemma 3.6, we have $\Gamma_{\mu}\left(J_{\Theta, \Pi}(V)\right)=\Gamma_{\mu}\left(\widehat{J}_{\Theta, \Pi}(V)\right)$. We also have

$$
\begin{aligned}
\Gamma_{\mu}\left(\overline{\mathfrak{n}}_{\Theta}^{k} J_{\Theta, \Pi}(V)\right)=\sum_{\mu^{\prime}+\mu^{\prime \prime}=\mu} \Gamma_{\mu^{\prime}}\left(\overline{\mathfrak{n}}_{\Theta}^{k}\right) \Gamma_{\mu^{\prime \prime}}\left(J_{\Theta, \Pi}(V)\right) \\
=\sum_{\mu^{\prime}+\mu^{\prime \prime}=\mu} \Gamma_{\mu^{\prime}}\left(\overline{\mathfrak{n}}_{\Theta}^{k}\right) \Gamma_{\mu^{\prime \prime}}\left(\widehat{J}_{\Theta, \Pi}(V)\right)=\Gamma_{\mu}\left(\overline{\mathfrak{n}}_{\Theta}^{k} \widehat{J}_{\Theta, \Pi}(V)\right) .
\end{aligned}
$$


We get (1).

(2) This follows from (1).

(3) By (2), it is sufficient to prove that for $V \in \mathcal{H C} \mathcal{C}_{\Theta}, \widehat{J}_{\Theta, \Pi}(V) \simeq \prod_{\mu \in \mathfrak{a}_{\Theta}^{*}} \Gamma_{\mu}(V)$. We can use the proof of GW80, Lemma 2.2].

(4) The kernel of $V \rightarrow \widehat{J}_{\Theta, \Pi}(V)$ satisfies $\operatorname{Ker} /\left(\mathfrak{m}_{\Theta} \cap \overline{\mathfrak{n}}_{\Theta}\right)$ Ker $=0$. Therefore, it is sufficient to prove that for $V \in \mathcal{H C}_{\Theta^{\prime}}$ with $V \neq 0, V /\left(\mathfrak{m}_{\Theta} \cap \overline{\mathfrak{n}}_{\Theta}\right) V \neq 0$. We

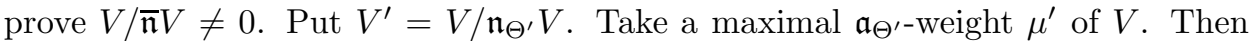
$\Gamma_{\mu^{\prime}}\left(\mathfrak{n}_{\Theta^{\prime}} V\right)=0$. Hence $\Gamma_{\mu^{\prime}}\left(V^{\prime}\right)=\Gamma_{\mu^{\prime}}(V)$. In particular, $V^{\prime} \neq 0$. By Lemma $3.2 V^{\prime}$ is a Harish-Chandra module of $L_{\Theta^{\prime}, \mathbb{R}}$. By Casselman's subrepresentation theorem, we have $V / \overline{\mathfrak{n}} V=V^{\prime} /\left(\mathfrak{m}_{\Theta^{\prime}} \cap \overline{\mathfrak{n}}\right) V^{\prime} \neq 0$.

Using (4), we regard $V$ as a submodule of $\widehat{J}_{\Theta, \Pi}(V)$. Let $d \nu: \mathbb{C} \rightarrow \mathfrak{a}$ be the differential of $\nu$ and put $H=d \nu(1)$. This is an integral dominant element of $\mathfrak{a}_{\Theta}$. In general, for an a-module $V$, let $\Gamma_{H, a}(V)$ be the generalized $H$-eigenspace of $V$ with an eigenvalue $a$. For $a, a^{\prime} \in \mathbb{C}$, we define $a \geq_{\mathbb{Z}} a^{\prime}$ by $a-a^{\prime} \in \mathbb{Z}_{\geq 0}$. For $V \in \mathcal{H C}_{\Theta^{\prime}, \rho}$ and $a \in \mathbb{C}$, define $F_{a}\left(\widehat{J}_{\Theta, \Pi}(V)\right) \subset \widehat{J}_{\Theta, \Pi}(V)$ and $F_{a} V \subset V$ by

$$
F_{a}\left(\widehat{J}_{\Theta, \Pi}(V)\right)=\prod_{a^{\prime} \leq \mathbb{Z} a} \Gamma_{H, a^{\prime}}\left(\widehat{J}_{\Theta, \Pi}(V)\right), \quad F_{a} V=V \cap F_{a}\left(\widehat{J}_{\Theta, \Pi}(V)\right) .
$$

Let $\mathscr{D}$ be the ring of differential operators on $G / B$. Set $\mathscr{V}=\mathscr{D} \otimes_{U(\mathfrak{g})} V, \widetilde{\mathscr{V}}=a_{\nu}^{*} \mathscr{V}$ and $\widetilde{V}=\Gamma\left(G / B \times \mathbb{G}_{m}, \widetilde{\mathscr{V}}\right)=\mathbb{C}\left[t, t^{-1}\right] \otimes V$. As in [ENV04, we define the filtration $V^{a}(\widetilde{V})$ on $\widetilde{V}$ by

$$
V^{a}(\widetilde{V})=\bigoplus_{k \in \mathbb{Z}} t^{k} F_{-a+k}(V)
$$

Lemma 4.4. We have

$$
F_{-a}(V) / F_{-a-1}(V) \stackrel{\sim}{\rightarrow} F_{-a}\left(\widehat{J}_{\Theta, \Pi}(V)\right) / F_{-a-1}\left(\widehat{J}_{\Theta, \Pi}(V)\right) \simeq \bigoplus_{\mu(H)=a} \Gamma_{\mu}\left(J_{\Theta, \Pi}(V)\right)
$$

Proof. We prove that the first homomorphism is isomorphic. By the definition of $F_{-a-1}(V)$, the homomorphism is injective. This homomorphism is surjective by Lemma 3.7 The second homomorphism is obviously an isomorphism.

From this lemma, if we prove that $V^{a}(\widetilde{V})$ is a $V$-filtration, then by the description of the nearby cycle functor in terms of $\mathscr{D}$-modules Kas83, (see [ENV04, 3]) and Lemma 3.6. we have $\Gamma(G / B, R \psi \widetilde{\mathscr{V}})=J_{\Theta, \Pi}(V)$. Hence Theorem 4.2 is proved.

To prove that this gives a $V$-filtration, it is sufficient to prove the following lemma. (See [ENV04, 4].) Define a filtration $F_{a}\left(U\left(\overline{\mathfrak{n}}_{\Theta}\right)\right)$ by

$$
F_{a}\left(U\left(\overline{\mathfrak{n}}_{\Theta}\right)\right)=\bigoplus_{a^{\prime} \leq \mathbb{Z} a} \Gamma_{H, a^{\prime}}\left(U\left(\overline{\mathfrak{n}}_{\Theta}\right)\right)
$$

Lemma 4.5. For $a \in \mathbb{C}$ and $k, l \in \mathbb{Z}$, the following hold.

(1) For a sufficiently large $k, F_{-a+k}(V)$ is stable.

(2) The module $F_{-a-k}(V) /\left(F_{-k}\left(U\left(\overline{\mathfrak{n}}_{\Theta}\right)\right) F_{-a}(V)\right)$ is a finitely generated $U\left(\mathfrak{m}_{\Theta}\right)$ module.

(3) For $l \geq 0$, we have $F_{-a-k-l}(V)=F_{-l}\left(U\left(\overline{\mathfrak{n}}_{\Theta}\right)\right) F_{-a-k}(V)$ for a sufficiently large $k$. 
Proof. (1) Take a finite subset $\Lambda \subset \mathfrak{a}_{\Theta}^{*}$ such that $\operatorname{wt}_{\mathfrak{a}_{\Theta}}\left(\widehat{J}_{\Theta, \Pi}(V)\right) \subset \Lambda-\left.\mathbb{Z}_{\geq 0} \Pi\right|_{\mathfrak{a}_{\Theta}}$. Since $H$ is dominant integral, for all $\mu \in \mathrm{wt}_{\mathfrak{a}_{\Theta}}\left(\widehat{J}_{\Theta, \Pi}(V)\right)$ we have $\mu^{\prime}(H)-\mu(H) \in$ $\mathbb{Z}_{\geq 0}$ for some $\mu^{\prime} \in \Lambda$. Take $k$ such that $-a+k \geq \max \left\{\mu^{\prime}(H) \mid \mu^{\prime} \in \Lambda\right\}$. For such $k, F_{-a+k}(V)$ is stable.

$(2,3)$ We can use the same proof as that of [ENV04, Lemma 2.5].

From this, $V^{a}(\widetilde{V})$ is a $V$-filtration. Hence we get Theorem 4.2

\section{Symmetric SPACE}

Assume that $G$ is of adjoint type. Let $\omega_{\alpha}$ be the fundamental coweight for $\alpha \in \Pi$, namely, it is a cocharacter $\omega_{\alpha}: \mathbb{G}_{m} \rightarrow G$ which satisfies $\left\langle\omega_{\alpha}, \alpha\right\rangle=1$ and $\left\langle\omega_{\alpha}, \beta\right\rangle=0$ for $\beta \in \Pi \backslash\{\alpha\}$. Since $G$ is of adjoint type, it exists. Define $\omega:\left(\mathbb{G}_{m}\right)^{\Pi} \rightarrow A$ by $\left(t_{\alpha}\right)_{\alpha} \mapsto \prod_{\alpha \in \Pi} \omega_{\alpha}\left(t_{\alpha}\right)$. Then $\omega$ gives an isomorphism.

De Concini and Procesi [DCP83] constructed the wonderful compactification $X$ of $G / K$. This compactification satisfies the following conditions. Set $x_{0}=K \in$ $G / K$.

(C1) The variety $X$ is irreducible and proper smooth over $\mathbb{C}$.

(C2) A $G$-orbit of $X$ is parameterized by a subset of $\Pi$. We denote the $G$-orbit corresponding to $\Theta \subset \Pi$ by $X_{\Theta}$.

(C3) The $G$-orbit $X_{\Pi}$ is the unique open $G$-orbit and it is isomorphic to $G / K$.

(C4) The closure of each orbit is smooth.

(C5) We have an $\bar{N}$-equivariant open embedding $\bar{N} \times \mathbb{A}^{\Pi} \rightarrow X$ such that for all $a=\left(a_{\alpha}\right) \in\left(\mathbb{G}_{m}\right)^{\Pi}$, an element $\omega(a) x_{0}$ is the image of $\left(1,\left(a_{\alpha}^{-2}\right)\right) \in \bar{N} \times \mathbb{A}^{\Pi}$. Moreover, the intersection of $X_{\Theta}$ and $\bar{N} \times \mathbb{A}^{\Pi}$ is given by $\bar{N} \times\left(\mathbb{G}_{m}\right)^{\Theta} \times$ $\{0\}^{\Pi \backslash \Theta}$.

(C6) By the above condition, $\bar{N} \times \mathbb{A}^{\Pi}$ is regarded as an open subvariety of $X$. Then the stabilizer of $\left(1,\left(1^{\Theta}, 0^{\Pi \backslash \Theta}\right)\right)$ in $G$ is $K_{\Theta} A_{\Theta} N_{\Theta}$.

Remark 5.1. The parameterization of $G$-orbits in [DCP83] is different from ours. In DCP83, the open orbit corresponds to $\emptyset$.

For each $\alpha \in \Pi$, put $Y_{\alpha}=\overline{X_{\Pi \backslash\{\alpha\}}}$. By the conditions (C4) and (C5), $\bigcup_{\alpha \in \Pi} Y_{\alpha}$ is a strict normal crossing divisor. Let $f: \mathcal{X} \rightarrow \mathbb{A}^{\Pi}$ be the variety constructed in Section 2 with respect to $\bigcup_{\alpha \in \Pi} Y_{\alpha}$. Each $Y_{\alpha}$ defines the subvariety $\mathcal{Y}_{\alpha} \subset \mathcal{X}$. Put $X^{\prime}=\bar{N} \times \mathbb{A}^{\Pi}$ and regard it as an open subvariety of $X$ by the condition (C5). Then $X^{\prime}$ defines an open subvariety $\mathcal{X}^{\prime}$ of $\mathcal{X}$. Since $Y_{\alpha} \cap X^{\prime}$ is isomorphic to $\left\{\left(\bar{n},\left(c_{\beta}\right)_{\beta \in \Pi}\right) \mid c_{\alpha}=0\right\}, \mathcal{X}^{\prime}$ is isomorphic to $\bar{N} \times \mathbb{A}^{\Pi} \times \mathbb{A}^{\Pi}$ by Lemma 2.4 (11).

Put $\mathcal{Z}=\mathcal{X} \backslash \bigcup_{\alpha \in \Pi} \mathcal{Y}_{\alpha}$ and $\mathcal{Z}^{\prime}=\mathcal{X}^{\prime} \cap \mathcal{Z}$. Let $f_{\mathcal{Z}}: \mathcal{Z} \rightarrow \mathbb{A}^{\Pi}$. Then we have $\mathcal{Z}^{\prime} \simeq \bar{N} \times\left(\mathbb{G}_{m}\right)^{\Pi} \times \mathbb{A}^{\Pi}$. Define a section $s: \mathbb{A}^{\Pi} \rightarrow \mathcal{Z}$ of $f_{\mathcal{Z}}: \mathcal{Z} \rightarrow \mathbb{A}^{\Pi}$ by $s(t)=$ $\left(1,1^{\Pi}, t\right) \in \bar{N} \times\left(\mathbb{G}_{m}\right)^{\Pi} \times \mathbb{A}^{\Pi} \simeq \mathcal{Z}^{\prime} \subset \mathcal{Z}$. For $\Theta \subset \Pi$, set $t_{\Theta}=\left(1^{\Theta}, 0^{\Pi \backslash \Theta}\right)$ and $x_{\Theta}=s\left(t_{\Theta}\right)$.

Lemma 5.2. For $\left(a_{\alpha}\right)_{\alpha} \in\left(\mathbb{G}_{m}\right)^{\Pi}$, the action of $\omega\left(a_{\alpha}\right) \in A$ on $\mathcal{X}^{\prime} \simeq \bar{N} \times \mathbb{A}^{\Pi} \times \mathbb{A}^{\Pi}$ is given by $(\bar{n}, d, t) \mapsto\left(\operatorname{Ad}\left(\omega\left(a_{\alpha}\right)\right) \bar{n},\left(a_{\alpha}^{-2}\right) d, t\right)$.

Proof. This follows from (C5) and Lemma 2.4 (3).

By Lemma 2.3, we have $\mathcal{X}_{\Theta} \simeq T_{\overline{X_{\Theta}}}(X) \times\left(\mathbb{G}_{m}\right)^{\Theta}$. For each subvariety $\mathcal{W} \subset \mathcal{X}$, put $\mathcal{W}_{\Theta}=\mathcal{W} \cap \mathcal{X}_{\Theta}$.

Lemma 5.3. The open subvariety $\mathcal{Z}_{\Theta} \subset \mathcal{X}_{\Theta}$ is contained in $T_{X_{\Theta}}(X) \times\left(\mathbb{G}_{m}\right)^{\Theta}$. 
Proof. By Lemma 2.3, we have $\mathcal{X}_{\Theta} \simeq T_{\overline{X_{\Theta}}}(X) \times\left(\mathbb{G}_{m}\right)^{\Theta}$ and $\left(\mathcal{Y}_{\alpha}\right)_{\Theta}=T_{Y_{\alpha} \cap \overline{X_{\Theta}}}\left(Y_{\alpha}\right) \times$ $\left(\mathbb{G}_{m}\right)^{\Theta}$. For $\alpha \in \Theta$, we have an obvious identity $\left.T_{\overline{X_{\Theta}}}(X)\right|_{Y_{\alpha} \cap \overline{X_{\Theta}}}=T_{Y_{\alpha} \cap \overline{X_{\Theta}}}\left(Y_{\alpha}\right)$. Thus we have $\left(\mathcal{X} \backslash \bigcup_{\alpha \in \Theta} \mathcal{Y}_{\alpha}\right)_{\Theta} \simeq T_{\overline{X_{\Theta}} \backslash \bigcup_{\alpha \in \Theta} Y_{\alpha}}(X) \times\left(\mathbb{G}_{m}\right)^{\Theta}=T_{X_{\Theta}}(X) \times\left(\mathbb{G}_{m}\right)^{\Theta}$. Hence $\mathcal{Z}_{\Theta} \subset T_{X_{\Theta}}(X) \times\left(\mathbb{G}_{m}\right)^{\Theta}$.

Lemma 5.4. The stabilizer of $x_{\Theta}$ in $G$ is $K_{\Theta} N_{\Theta}$.

Proof. Consider the morphism $\mathcal{Z}_{\Theta} \hookrightarrow T_{X_{\Theta}}(X) \times\left(\mathbb{G}_{m}\right)^{\Theta} \rightarrow T_{X_{\Theta}}(X) \rightarrow X_{\Theta}$. Let $y_{\Theta}$ be the image of $x_{\Theta}$. Then $y_{\Theta}=\left(1,\left(1^{\Theta}, 0^{\Pi \backslash \Theta}\right)\right) \in \bar{N} \times \mathbb{A}^{\Pi}=X^{\prime}$ by Lemma 2.4 (2). Hence $\operatorname{Stab}_{G}\left(x_{\Theta}\right) \subset \operatorname{Stab}_{G}\left(y_{\Theta}\right)=K_{\Theta} A_{\Theta} N_{\Theta}$ by (C6).

We prove $K_{\Theta} N_{\Theta} \subset \operatorname{Stab}_{G}\left(x_{\Theta}\right)$. By Lemma 2.3] we have $\mathcal{X}_{\Pi} \simeq X \times\left(\mathbb{G}_{m}\right)^{\Pi}$. Then $s\left(t^{2}\right)$ is given by $\left(\omega(t)^{-1} x_{0}, t^{2}\right) \in X \times\left(\mathbb{G}_{m}\right)^{\Pi}$ for $t \in\left(\mathbb{G}_{m}\right)^{\Pi}$ (cf. Lemma $2.4(1))$. Hence $\operatorname{Stab}_{G}\left(s\left(t^{2}\right)\right)=\operatorname{Ad}\left(\omega(t)^{-1}\right) K$. Its Lie algebra is spanned by $\mathfrak{m}$ and $\left\{\operatorname{Ad}\left(\omega(t)^{-1}\right)(X+\theta(X)) \mid X \in \mathfrak{g}_{\beta}, \beta \in \Sigma^{+}\right\}$. Here, $\mathfrak{g}_{\beta}$ is the root space for $\beta$. Since $\operatorname{Ad}\left(\omega(t)^{-1}\right)(X+\theta(X))=\beta\left(\omega(t)^{-1}\right)\left(X+\beta(\omega(t))^{2} \theta(X)\right)$, the Lie algebra of $\operatorname{Stab}_{G}\left(s\left(t^{2}\right)\right)$ is spanned by $\mathfrak{m}$ and $\left\{X+\beta(\omega(t))^{2} \theta(X) \mid X \in \mathfrak{g}_{\beta}, \beta \in \Sigma^{+}\right\}$. If $\beta=\sum_{\alpha \in \Pi} n_{\alpha} \alpha$, then $\beta(\omega(t))^{2}=\prod_{\alpha \in \Pi} t_{\alpha}^{2 n_{\alpha}}$ for $t=\left(t_{\alpha}\right) \in\left(\mathbb{G}_{m}\right)^{\Pi}$. Since this can be extended to any $t \in \mathbb{A}^{\Pi}$, the Lie algebra of $\operatorname{Stab}_{G}\left(s\left(t^{2}\right)\right)$ contains the space spanned by $\mathfrak{m}$ and $\left\{X+\beta(\omega(t))^{2} \theta(X) \mid X \in \mathfrak{g}_{\beta}, \beta \in \Sigma^{+}\right\}$for any $t \in \mathbb{A}^{\Pi}$.

Now set $t=t_{\Theta}$. Then $s\left(t^{2}\right)=s(t)=x_{\Theta}$. For $\beta=\sum_{\alpha \in \Pi} n_{\alpha} \alpha \in \Sigma^{+}, \beta(\omega(t))^{2}$ is 0 or 1 and it is 1 if and only if $n_{\alpha}=0$ for any $\alpha \in \Pi \backslash \Theta$, namely, $\mathfrak{g}_{\beta} \subset \mathfrak{m}_{\Theta}$. Hence the Lie algebra of $\operatorname{Stab}_{G}\left(x_{\Theta}\right)$ contains $\operatorname{Lie}\left(K_{\Theta} N_{\Theta}\right)$. Therefore, $\operatorname{Stab}_{G}\left(x_{\Theta}\right) \supset\left(K_{\Theta}\right)^{\circ} N_{\Theta}$. Since $K=M K^{\circ}$ and $M$ stabilizes $s(t)$ for all $t \in\left(\mathbb{G}_{m}\right)^{\Pi}$ (hence for all $t \in \mathbb{A}^{\Pi}$ ), we have $\operatorname{Stab}_{G}\left(x_{\Theta}\right) \supset M\left(K_{\Theta}\right)^{\circ} N_{\Theta}=K_{\Theta} N_{\Theta}$.

Finally, we prove that $\operatorname{Stab}_{A_{\Theta}}\left(x_{\Theta}\right) \subset M$. Since $M \subset K_{\Theta}$, this implies the lemma. For $\left(a_{\alpha}\right) \in\left(\mathbb{G}_{m}\right)^{\Pi}$, we have $\omega\left(a_{\alpha}\right) x_{\Theta}=\left(1,\left(a_{\alpha}^{-2}\right), t_{\Theta}\right) \in \bar{N} \times \mathbb{A}^{\Pi} \times \mathbb{A}^{\Pi} \simeq \mathcal{X}^{\prime}$. Hence if $\omega\left(a_{\alpha}\right) \in \operatorname{Stab}_{A_{\Theta}}\left(x_{\Theta}\right)$, then $a_{\alpha}^{2}=1$ for all $\alpha \in \Pi$. Therefore, $\omega\left(a_{\alpha}\right) \in M$.

Lemma 5.5. We have $G x_{\Theta}=f_{\mathcal{Z}}^{-1}\left(t_{\Theta}\right)$.

Proof. We regard $T_{X_{\Theta}}(X)$ as a subvariety of $\mathcal{X}$ by $T_{X_{\Theta}}(X)=T_{X_{\Theta}}(X) \times\left\{1^{\Theta}\right\} \subset$ $T_{X_{\Theta}}(X) \times\left(\mathbb{G}_{m}\right)^{\Theta} \cong \mathcal{X}_{\Theta} \subset \mathcal{X}$. By Lemma [5.3, we have $f_{\mathcal{Z}}^{-1}\left(t_{\Theta}\right)=T_{X_{\Theta}}(X) \cap \mathcal{Z}$. Let $p: T_{X_{\Theta}}(X) \rightarrow X_{\Theta}$ be the projection. Then $p\left(x_{\Theta}\right)=y_{\Theta}$, where $y_{\Theta}$ is given in the proof of the previous lemma. Since $X_{\Theta}$ is a $G$-orbit, we have $G y_{\Theta}=X_{\Theta}$. Hence it is sufficient to prove that $\operatorname{Stab}_{G}\left(y_{\Theta}\right) x_{\Theta}=p^{-1}\left(y_{\Theta}\right) \cap \mathcal{Z}$. By (C6) and Lemma 5.4 it is equivalent to showing that $A_{\Theta} x_{\Theta}=p^{-1}\left(y_{\Theta}\right) \cap \mathcal{Z}$. By Lemma 2.4 (2), we have $p^{-1}\left(y_{\Theta}\right)=\{1\} \times\left\{1^{\Theta}\right\} \times \mathbb{A}^{\Pi \backslash \Theta} \times\left\{\left(1^{\Theta}, 0^{\Pi \backslash \Theta}\right)\right\} \subset \bar{N} \times\left(\mathbb{G}_{m}\right)^{\Pi} \times \mathbb{A}^{\Pi}=\mathcal{X}^{\prime}$. Hence the lemma follows from Lemma 5.2 .

In general, for an algebraic group $H$ and an $H$-variety $Y$, let $\operatorname{Perv}_{H}(Y)$ be the category of $H$-equivariant perverse sheaves. Then $\mathcal{H C}_{\Theta, \rho} \simeq \operatorname{Perv}_{K_{\Theta} N_{\Theta}}(G / B) \simeq$ $\operatorname{Perv}_{K_{\Theta} N_{\Theta} \times B}(G) \simeq \operatorname{Perv}_{B}\left(G / K_{\Theta} N_{\Theta}\right)$. Write $\Delta_{\Theta}^{\prime}$ for this equivalence. Let $\Theta_{2} \subset$ $\Theta_{1} \subset$ П. Take $n_{\alpha} \in \mathbb{Z}_{\geq 1}$ for each $\alpha \in \Theta_{1} \backslash \Theta_{2}$ and define $\nu: \mathbb{A}^{1} \rightarrow \mathbb{A}^{\Pi}$ by $\nu(t)=\left(t^{n_{\alpha}}\right)_{\alpha \in \Theta_{1} \backslash \Theta_{2}} \times\left(0^{\Pi \backslash \Theta_{1}}\right) \times\left(1^{\Theta_{2}}\right)$. Put $\mathcal{Z}_{\nu}=\mathcal{Z} \times_{\mathbb{A}^{\Pi}} \mathbb{A}^{1}$ and denote the canonical morphism $\mathcal{Z}_{\nu} \rightarrow \mathbb{A}^{1}$ by $f_{\nu}$. Then, by Lemma 5.4 and Lemma 5.5 we have $f_{\nu}^{-1}(0) \simeq$ $G / K_{\Theta_{2}} N_{\Theta_{2}}$ and $f_{\nu}^{-1}\left(\mathbb{G}_{m}\right) \simeq G / K_{\Theta_{1}} N_{\Theta_{1}} \times \mathbb{G}_{m}$. Let $p_{\nu}: f_{\nu}^{-1}\left(\mathbb{G}_{m}\right) \simeq G / K_{\Theta_{1}} N_{\Theta_{1}} \times$ $\mathbb{G}_{m} \rightarrow G / K_{\Theta_{1}} N_{\Theta_{1}}$ be the first projection and $R \psi$ be the nearby cycle functor with respect to $f_{\nu}$. Define $\operatorname{Kat}_{\nu}: \operatorname{Perv}_{B}\left(G / K_{\Theta_{1}} N_{\Theta_{1}}\right) \rightarrow \operatorname{Perv}_{B}\left(G / K_{\Theta_{2}} N_{\Theta_{2}}\right)$ by $\mathrm{Kat}_{\nu}=R \psi \circ p_{\nu}^{*}$.

Now we prove the main theorem of this paper. 
Theorem 5.6. As functors $\mathcal{H C}_{\Theta_{1}, \rho} \rightarrow \operatorname{Perv}_{B}\left(G / K_{\Theta_{2}} N_{\Theta_{2}}\right)$, we have $\operatorname{Kat}_{\nu} \circ \Delta_{\Theta_{1}}^{\prime} \simeq$ $\Delta_{\Theta_{2}}^{\prime} \circ J_{\Theta_{2}, \Theta_{1}}$.

Proof. Let $s_{\nu}: \mathbb{A}^{1} \rightarrow \mathcal{Z}_{\nu}$ be the section of $f_{\nu}$ obtained by the base change of $s$ under $\nu$. Consider the following diagram:

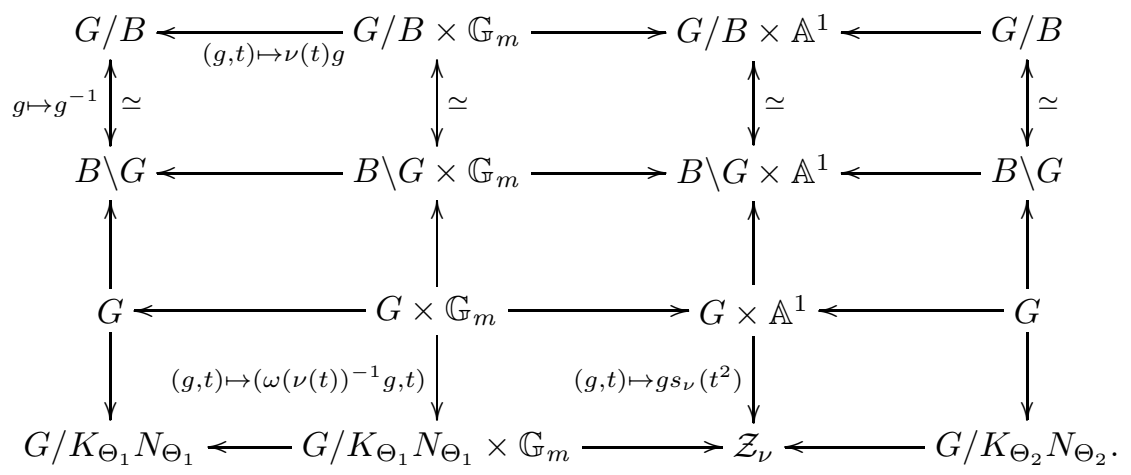

Every rectangle in the diagram above is cartesian, and every vertical arrow is smooth. The functor $\Psi_{\nu}$ of Emerton-Nadler-Vilonen is defined as the nearby cycle functor with respect to the top row, and the functor $\mathrm{Kat}_{\nu}$ is defined as the nearby cycle functor with respect to the bottom row. Therefore, we get our theorem by Theorem 4.2 and the smooth base change theorem.

\section{REFERENCES}

[BB81] A. Bẹlinson and J. Bernstein, Localisation de g-modules, C. R. Acad. Sci. Paris Sér. I Math. 292 (1981), no. 1, 15-18.

[Cas80] W. Casselman, Jacquet modules for real reductive groups, Proceedings of the International Congress of Mathematicians (Helsinki, 1978) (Helsinki), Acad. Sci. Fennica, 1980, pp. 557-563.

[CO78] W. Casselman and M. S. Osborne, The restriction of admissible representations to $\mathfrak{n}$, Math. Ann. 233 (1978), no. 3, 193-198.

[DCP83] C. De Concini and C. Procesi, Complete symmetric varieties, Invariant theory (Montecatini, 1982), Lecture Notes in Math., vol. 996, Springer, Berlin, 1983, pp. 1-44.

[ENV04] M. Emerton, D. Nadler, and K. Vilonen, A geometric Jacquet functor, Duke Math. J. 125 (2004), no. 2, 267-278.

[Fu198] W. Fulton, Intersection theory, second ed., Ergebnisse der Mathematik und ihrer Grenzgebiete. 3. Folge. A Series of Modern Surveys in Mathematics, vol. 2, SpringerVerlag, Berlin, 1998.

[GW80] R. Goodman and N. R. Wallach, Whittaker vectors and conical vectors, J. Funct. Anal. 39 (1980), no. 2, 199-279.

[HS83a] H. Hecht and W. Schmid, Characters, asymptotics and $\mathfrak{n}$-homology of Harish-Chandra modules, Acta Math. 151 (1983), no. 1-2, 49-151.

[HS83b] H. Hecht and W. Schmid, On the asymptotics of Harish-Chandra modules, J. Reine Angew. Math. 343 (1983), 169-183.

[Kas83] M. Kashiwara, Vanishing cycle sheaves and holonomic systems of differential equations, Algebraic geometry (Tokyo/Kyoto, 1982), Lecture Notes in Math., vol. 1016, Springer, Berlin, 1983, pp. 134-142.

$\left[\mathrm{KKM}^{+} 78\right]$ M. Kashiwara, A. Kowata, K. Minemura, K. Okamoto, T. Oshima, and M. Tanaka, Eigenfunctions of invariant differential operators on a symmetric space, Ann. of Math. (2) 107 (1978), no. 1, 1-39.

[SS97] P. Schneider and U. Stuhler, Representation theory and sheaves on the Bruhat-Tits building, Inst. Hautes Études Sci. Publ. Math. (1997), no. 85, 97-191.

[Wal88] N. R. Wallach, Real reductive groups. I, Pure and Applied Mathematics, vol. 132, Academic Press Inc., Boston, MA, 1988. 
(Noriyuki Abe) Creative Research Institution, Hokkaido University, N21 W10, KitaKU SAPPORO 001-0021, JAPAN

E-mail address: abenori@math.sci.hokudai.ac.jp

(Yoichi Mieda) Faculty of Mathematics, Kyushu University, 744 Motooka, Nishi-Ku, FUKUOKA, 819-0395 JAPAN

E-mail address: mieda@math.kyushu-u.ac.jp 